Prepared FOR SUBMission TO JCAP

\title{
The impact of the phase-space density on the indirect detection of dark matter
}

\author{
Francesc Ferrer, Daniel R. Hunter \\ Physics Department and McDonnell Center for the Space Sciences \\ Washington University, St Louis, MO 63130, USA
}

\begin{abstract}
We study the indirect detection of dark matter when the local dark matter velocity distribution depends upon position, as expected for the Milky Way and its dwarf spheroidal satellites, and the annihilation cross-section is not purely s-wave. Using a phasespace distribution consistent with the dark matter density profile, we present estimates of cosmic and gamma-ray fluxes from dark matter annihilations. The expectations for the indirect detection of dark matter can differ significantly from the usual calculation that assumes that the velocity of the dark matter particles follows a Maxwell-Boltzmann distribution.
\end{abstract}




\section{Contents}

1 Introduction $\quad 1$

2 The dark matter annihilation rate $\quad 3$

3 Density profile of the dark matter halo $\quad 5$

$\begin{array}{lll}3.1 & \text { NFW profile } & 6\end{array}$

$\begin{array}{lll}3.2 & \text { Einasto profile } & 7\end{array}$

3.3 Burkert profile 8

$\begin{array}{lll}3.4 & \text { Galactic bulge and disk } & 8\end{array}$

4 Phase-space distribution function $\quad 9$

4.1 The relative velocity distribution $\quad 11$

4.2 Consistency with Jeans analysis $\quad 15$

5 Fluxes from dark matter annihilations $\quad 17$

$\begin{array}{ll}5.1 \text { Sommerfeld enhancement } & 20\end{array}$

6 Results and discussion $\quad 21$

7 Conclusion $\quad 26$

$\begin{array}{ll}\text { A Relative velocity distribution for an ergodic system } & 27\end{array}$

\section{Introduction}

Besides dark matter's gravitational effects, which establishes it as the second largest contributor to the energy budget of the Universe, very little is known about its fundamental nature. A popular, and well-motivated, candidate for composing the dark matter is a weakly interacting massive particle (WIMP). With mass and interactions at the electro-weak energy scale, WIMPs naturally obtain a thermal relic density in the range required by cosmological observations. The foremost example of WIMP is the lightest neutral supersymmetric particle, the neutralino [1].

Importantly, weak-scale interactions with Standard Model particles make it possible, in principle, to detect the presence of WIMPs. Direct detection experiments monitor the recoil of nuclei that might be elastically scattered by dark matter particles. On the other hand, WIMPs can self-annihilate and produce gamma-rays, neutrinos, or other Standard Model particles. This is the basis for the indirect detection of dark matter [1,2].

Over the past few years, direct detection experiments have attained the sensitivity to probe the parameter space of the simplest supersymmetric extensions of the Standard Model (SM), and next-generation multi-ton experiments [3] are being planned to test less constrained extensions [4, 5]. Even though, motivated by theoretical expectations on the neutralino, experimental efforts have focused on the DM mass range $100 \mathrm{GeV} \lesssim m_{\chi} \lesssim 1 \mathrm{TeV}$, several experiments have reported signals that can be attributed to low-mass dark matter particles, $m_{\chi} \lesssim 10 \mathrm{GeV}$ [6-8]. The strong tension of this interpretation with the constraints set by the null results of other low-background experiments, such as XENON100 and CDMS-Ge [9, 10], 
can be eased, although not completely eliminated [11], by considering dark matter particles with non-standard interactions [12-14] and systematic errors in the determination of the detector response at low recoil energies $[15,16]$. The interpretation of the data depends also on the assumed velocity distribution of the DM halo [17-20], and this has prompted investigators to consider more realistic scenarios than the Standard Halo with a MaxwellBoltzmann distribution [21-26].

Similarly, several tantalizing observations of cosmic and gamma-ray fluxes have been linked to the annihilation or decay of DM particles. The bright $511 \mathrm{keV}$ line emission from the bulge of the Galaxy detected by the SPI spectrometer on the INTEGRAL satellite [27], the excesses of microwaves and gamma-rays in the inner Galaxy revealed by the WMAP and Fermi satellites [28], the evidence for a $130 \mathrm{GeV}$ spectral line in the Fermi data [29, 30], and the rise in the positron fraction above $10 \mathrm{GeV}$ observed by PAMELA and AMS-02 [31, 32], have been attributed to the effects of DM [33-37].

In addition to spectral features, the fact that the signals in the photon sector originate in the Galactic center bodes well for the DM interpretation. Indeed, the fluxes from DM annihilations depend on the square of the density, and N-body simulations suggest that DM halos are cusped at the center [38]. However, DM particles must have non-standard interactions to account for these observations. An appealing feature of the WIMP miracle is that the cross-section that enters the predictions for the indirect detection is directly related to the thermal cross-section, $\sigma v \sim 3 \times 10^{-26} \mathrm{~cm}^{3} / \mathrm{s}$, that explains the observed cosmological abundance of DM. Since the DM particles were relativistic at decoupling but are moving at non-relativistic speeds in the halo of the Galaxy, a $p$-wave velocity dependent flux, $\sigma v \sim b v^{2}$, can result in the right relic density for a light $\mathrm{MeV}$ dark matter particle that annihilates into non-relativistic $e^{ \pm}$pairs with $\sigma v \sim 10^{-5} \mathrm{pb}$, giving rise to the observed $511 \mathrm{keV}$ emission from the bulge [39, 40].

Similarly, barring the presence of a nearby DM clump [41, 42], the thermal cross-section falls short by about two orders of magnitude to explain the AMS-02 data. Here, the correct relic abundance and a larger annihilation cross-section can be reconciled if the annihilation follows $\sigma v \propto 1 / v$. This so-called Sommerfeld enhancement results from the exchange of light particles, and is at the basis for the DM explanation of the rising positron fraction at $\mathrm{GeV}$ energies [43-46]. These scenarios face stringent constraints from anti-proton, gamma-ray and synchrotron data, and there is some tension with the higher DM density in the galactic center predicted by the steep NFW and Einasto profiles favored by N-body simulations [47-51].

In the simplest WIMP models the annihilation flux is mostly $s$-wave, and independent of the velocity, $\sigma v \sim a$. As mentioned above, then the flux from DM annihilations varies through the halo tracking the square of the density, which is expected to be larger at the center of the halo or in the dwarf Spheroidal satellites of the Milky Way [52]. When the annihilation is velocity dependent, however, the flux is also affected by the distribution of DM particle velocities, which depends on the location in the halo. For instance, for the same density profile, a p-wave annihilating DM gives a shallower distribution of the halo flux [53] and results in a different power spectrum for the diffuse cosmological signal [54]. In the case of Sommerfeld enhancement, the flux is greatly increased towards the center of the halo due to smaller DM velocities [55].

In this paper we take a closer look at the distribution of the annihilation rate of DM particles in the halo for a general velocity dependent cross-section. Previous works applied the Jeans equation to estimate the variance in the velocity within the halo, which was then used as a proxy for the relative velocity of the annihilating DM particles. Although this is enough 
for p-wave DM, it does not take into account kurtosis and other deviations from Gaussianity in the velocity distribution that might be important for Sommerfeld enhanced and more general models. After reviewing the calculation of the DM annihilation rate in Sec. 2, we list the different DM profiles that we use to describe galactic and dSph sized haloes in Sec. 3. An important aspect of our treatment is that we include the effect of baryons that dominate the gravitational potential in the central regions of the Galaxy. For each halo we find a corresponding single particle velocity distribution using Eddington's formula in Sec. 4, which we then use to derive the relative velocity distribution. Sec. 5 contains our calculation of the DM annihilation rates, which turn out to be larger than previous estimates based on the Jeans equation. As discussed in Sec. 6, this aggravates the tension between an interpretation of the rising positron fraction based on DM annihilations and the constraints from gamma-ray and synchrotron data.

\section{The dark matter annihilation rate}

A pair of dark matter particles, $\chi$, may annihilate into a final state consisting of Standard Model particles with a probability per unit time

$$
\mathrm{d} \Gamma_{2 \chi}=\mathrm{d} \sigma \times \Phi_{2 \chi}
$$

Here, $\mathrm{d} \sigma$ is the differential cross-section for the annihilation process, and $\Phi_{2 \chi}$ is the flux of either initial particle at the position of the other one defined as the product of the number density $n_{\chi}$ and the relative velocity $u_{\chi}$ :

$$
\Phi_{2 \chi}=u_{\chi} n_{\chi}=u_{\chi} \frac{\rho_{\chi}}{m_{\chi}},
$$

where $\rho_{\chi}$ is the DM density. In a frame where the annihilating dark matter particles have four-momenta $p_{i}=\left(E_{i}, \boldsymbol{p}_{i}\right), i=1,2$, the relative velocity takes the value

$$
u_{\chi}=\frac{\sqrt{\left(p_{1} \cdot p_{2}\right)^{2}-m_{\chi}^{4}}}{E_{1} E_{2}} .
$$

In the center of mass frame, $\boldsymbol{p}_{1}=-\boldsymbol{p}_{2}=\boldsymbol{p}$, and eq. (2.3) reduces to $u_{\chi}=\left|\frac{\boldsymbol{p}}{E_{1}}-\frac{-\boldsymbol{p}}{E_{2}}\right|=$ $\left|\boldsymbol{v}_{1}-\boldsymbol{v}_{2}\right|$. When the two annihilating particles are identical, $E_{1}=E_{2}$ in the center of mass frame, and $u_{\chi}=2|\boldsymbol{v}|$, which is the magnitude of the relative velocity, $v_{\text {rel }}{ }^{1}$

The density $n_{\chi}$ will depend on the position in the halo as described below. On the other hand, the differential cross-section depends on the momenta of the initial and final particles, and on the particle physics model, but not on the spatial coordinates. ${ }^{2}$ The rate of particles of type $j$ that are generated in a volume element $\mathrm{d} V$ at the position $\boldsymbol{x}$ in the halo, containing $n_{\chi}(\boldsymbol{x}) \mathrm{d} V$ dark matter particles, is:

$$
\frac{\mathrm{d}^{2} \Gamma}{\mathrm{d} E \mathrm{~d} V}=n_{\chi}(\boldsymbol{x}) \sum_{i} B R_{i} \frac{\mathrm{d} N_{j, i}}{\mathrm{~d} E}\left\langle\sigma_{i} \times \Phi_{2 \chi}\right\rangle_{\boldsymbol{x}}
$$

\footnotetext{
${ }^{1}$ However, in this frame $u_{\chi}$ is not really a physical velocity, since it can take values as large as 2 for extremely relativistic particles.

${ }^{2}$ We do not consider environmental effects, such as screening mechanisms, that could play a role in the dark energy sector (see e.g. [56] for a review).
} 
where $B R_{i}$ is the branching ratio for the reaction $i$, that produces an average of $\mathrm{d} N_{j, i}$ particles of type $j$ with energies between $E$ and $E+\mathrm{d} E$, and $\langle$.$\rangle is the average over all possible initial$ kinematic configurations of the DM particles.

Though dark matter particles had relativistic energies in the early universe [57], e.g. around the time of decoupling and freeze-out, the annihilating particles in the weak gravitational field of the galactic halo are moving at non-relativistic speeds, $v \sim 10^{-3} c$, and can be fully described by a Newtonian ${ }^{3}$ distribution function (DF) $f$ such that $f(\boldsymbol{x}, \boldsymbol{v}, t) \mathrm{d}^{3} \boldsymbol{x} \mathrm{d}^{3} \boldsymbol{v}$ is the probability that a particle $\chi$ has phase-space coordinates in the given range at time $t[60]$. We will be only concerned with steady-state systems, and we drop the explicit time dependence in the DF. Also, since some popular dark matter density profiles do not have a well-defined finite total mass, unless they are truncated, it is convenient to redefine the DF so that it corresponds to the mass density in phase-space.

The dark matter density in eq. (2.2) can be recovered from the DF by marginalizing over velocities

$$
\rho_{\chi}(\boldsymbol{x}) \equiv \int \mathrm{d}^{3} \boldsymbol{v} f(\boldsymbol{x}, \boldsymbol{v})
$$

The dark matter particles at the point $\boldsymbol{x}$ in the halo have velocities following the distribution

$$
P_{\boldsymbol{x}}(\boldsymbol{v})=\frac{f(\boldsymbol{x}, \boldsymbol{v})}{\rho(\boldsymbol{x})}
$$

which is properly normalized to one. We can then explicitly write the average over initial configurations in eq. (2.4) as:

$$
\frac{\mathrm{d}^{2} \Gamma}{\mathrm{d} E \mathrm{~d} V}=n_{\chi}(\boldsymbol{x}) \sum_{i} B R_{i} \frac{\mathrm{d} N_{j, i}}{\mathrm{~d} E} \int \mathrm{d}^{3} \boldsymbol{v}_{1} \mathrm{~d}^{3} \boldsymbol{v}_{2} P_{\boldsymbol{x}}\left(\boldsymbol{v}_{1}\right) P_{\boldsymbol{x}}\left(\boldsymbol{v}_{2}\right) \sigma_{i} \times \Phi_{2 \chi} .
$$

We can further simplify this expression in the center of mass frame of the annihilating particles by introducing the relative velocity distribution. Given the velocity distribution for a single particle as found above, we can find the relative velocity distribution in the center of mass frame of the annihilating particles by noting that the probability of two particles having velocities $\boldsymbol{v}_{1}$ and $\boldsymbol{v}_{2}$ must be equal to the probability of a pair of particles having center of mass velocity $\boldsymbol{v}_{\mathrm{cm}}=\left(\boldsymbol{v}_{1}+\boldsymbol{v}_{2}\right) / 2$ and relative velocity $\boldsymbol{v}_{\text {rel }}=\boldsymbol{v}_{1}-\boldsymbol{v}_{2}$, or, in terms of the individual velocity distribution, eq. (2.6),

$$
\begin{aligned}
P_{\boldsymbol{x}}\left(\boldsymbol{v}_{1}\right) P_{\boldsymbol{x}}\left(\boldsymbol{v}_{2}\right) \mathrm{d}^{3} \boldsymbol{v}_{1} \mathrm{~d}^{3} \boldsymbol{v}_{2} & =P_{\boldsymbol{x}}\left(\boldsymbol{v}_{\mathrm{cm}}+\boldsymbol{v}_{\mathrm{rel}} / 2\right) P_{\boldsymbol{x}}\left(\boldsymbol{v}_{\mathrm{cm}}-\boldsymbol{v}_{\mathrm{rel}} / 2\right) \mathrm{d}^{3} \boldsymbol{v}_{\mathrm{cm}} \mathrm{d}^{3} \boldsymbol{v}_{\mathrm{rel}} \\
& \equiv P_{\boldsymbol{x}, \text { pair }}\left(\boldsymbol{v}_{\mathrm{cm}}, \boldsymbol{v}_{\mathrm{rel}}\right) \mathrm{d}^{3} \boldsymbol{v}_{\mathrm{cm}} \mathrm{d}^{3} \boldsymbol{v}_{\mathrm{rel}} .
\end{aligned}
$$

Integrating over the center of mass velocity, since the annihilation process is invariant under translations, and writing eq. (2.1) in the center of mass frame, we obtain a general expression for the production rate of particles of type $j$ :

$$
\frac{\mathrm{d}^{2} \Gamma}{\mathrm{d} E \mathrm{~d} V}=n_{\chi}^{2}(\boldsymbol{x}) \sum_{i} B R_{i} \frac{\mathrm{d} N_{j, i}}{\mathrm{~d} E} \int \mathrm{d}^{3} \boldsymbol{v}_{\text {rel }} P_{\boldsymbol{x}, \text { rel }}\left(\boldsymbol{v}_{\mathrm{rel}}\right) \sigma_{i} v_{\mathrm{rel}},
$$

where the we have defined the relative velocity distribution:

$$
P_{\boldsymbol{x}, \text { rel }}\left(\boldsymbol{v}_{\mathrm{rel}}\right) \equiv \int P_{\boldsymbol{x}, \text { pair }}\left(\boldsymbol{v}_{\mathrm{cm}}, \boldsymbol{v}_{\mathrm{rel}}\right) \mathrm{d}^{3} \boldsymbol{v}_{\mathrm{cm}}
$$

\footnotetext{
${ }^{3}$ Except in the region close to the central black hole $[58,59]$.
} 
Furthermore, Lorentz invariance requires that the annihilation does not depend on the orientation of the relative velocity. Hence, to obtain the average annihilation rate we just need to convolve the cross-section with the distribution of the magnitude of the relative velocity.

\section{Density profile of the dark matter halo}

The possibility to detect dark matter particles in the halo was initially studied assuming that their distribution was that of the Standard Halo [61], which models the galaxy after a singular isothermal sphere profile:

$$
\rho_{S I S}(r)=\frac{\sigma^{2}}{2 \pi G r^{2}}
$$

The increasing resolution of numerical N-body simulations in recent years, however, has resulted in different density profiles that provide a better description of the dark matter halo (see e.g. [38, 62, 63] for recent reviews). While less singular than eq. (3.1), the predictions from numerical experiments suggest cuspy densities rising as $\sim 1 / r$ towards the center. Several astrophysical observations, however, have shown that distributions with a constant density core might provide a more accurate description of smaller halos, such as satellite galaxies of the Milky Way, or of the galactic center (see e.g. [64] for a review of observations of LSB and gas-rich galaxies). Hence, we will consider both cored and cusped halos to model the spatial distribution of the dark matter particles.

Although there is evidence from numerical simulations for the existence of substructure in the form of unmixed dark matter clumps, we are primarily concerned with annihilations from the smooth halo, which we take to be spherically symmetric. As we argue in the discussion section, these approximations provide a conservative estimate of the dark matter annihilation rate.

We find it advantageous to work with dimensionless densities and potentials. Dehnen profiles [65], falling as $1 / r^{4}$ at large distances, have a well defined total mass. This is not the case, however, for the NFW [66] profile, whose mass grows logarithmically at large distances. One then usually defines a virial radius $r_{\text {vir }}$ such that the average density contained in the spherical volume $4 / 3 \pi r_{\text {vir }}^{3}$ is $\delta_{c} \approx 200$ times the critical density of the universe, $\rho_{\text {crit }}$. We will use the mass $M_{\text {vir }}$ contained in this region to normalize gravitational potentials and densities, and we measure lengths in units of $r_{\mathrm{vir}}$ :

$$
x=\frac{r}{r_{\mathrm{vir}}} .
$$

We consider spherically symmetric DM density profiles of the form

$$
\rho=\rho_{0} \tilde{\rho}(x, c, \cdots),
$$

where $\tilde{\rho}$ is dimensionless. Here, $c \equiv r_{\text {vir }} / a$ is the concentration parameter, with $a$ being a typical length scale of the halo. For example, in two-power density models $a$ is the intermediate radius that marks the smooth transition between the inner (possibly cuspy) power-law and the $1 / r^{3-4}$ behaviour at large radii. For cored profiles, $a$ could represent the size of a central finite density region, and there might be additional parameters in more complicated multi-scale models, which we will omit from here on. 
For a given profile,

$$
M_{\mathrm{vir}}=4 \pi r_{\mathrm{vir}}^{3} \rho_{0} \underbrace{\int_{0}^{1} x^{2} \tilde{\rho}(x, c) \mathrm{d} x}_{\equiv g(c)}
$$

and we can rewrite eq. (3.3) as:

$$
\rho=\frac{M_{\mathrm{vir}}}{4 \pi r_{\mathrm{vir}}^{3}} \frac{1}{g(c)} \times \tilde{\rho}(x, c) .
$$

We will make frequent use of dimensionless analogues of the relative potential $\psi \equiv-\Phi$, where $\Phi$ is the gravitational potential per unit mass, and the relative energy $\epsilon=\psi-v^{2} / 2$ :

$$
\begin{aligned}
\tilde{\epsilon} & \equiv \frac{r_{\mathrm{vir}}}{G M_{\mathrm{vir}}} \epsilon \\
\tilde{\psi} & \equiv \frac{r_{\mathrm{vir}}}{G M_{\mathrm{vir}}} \psi,
\end{aligned}
$$

where $G$ is Newton's gravitational constant.

The escape velocity at any given point of the halo is given by,

$$
v^{\max }(x)=\sqrt{2 \tilde{\psi}(x) \frac{G M_{\mathrm{vir}}}{r_{\mathrm{vir}}}} .
$$

Since the potential, $\tilde{\psi}$, attains its maximum at $x=0$, the escape velocity will be largest at the center of the halo.

The particular shape of the density profile can be inferred from observations of stars tracing the gravitational potential of the halo or from numerical N-body simulations. Although simulations favor a universal cusped profile, some sub-galactic sized objects are better described by assuming the presence of a central core. We, hence, consider both cusped and cored distributions.

\subsection{NFW profile}

The Navarro, Frenk, and White (NFW) profile,

$$
\begin{aligned}
\rho_{\mathrm{NFW}} & =\frac{\rho_{0}}{\frac{r}{a}\left(1+\frac{r}{a}\right)^{2}} \\
& =\frac{M_{\mathrm{vir}}}{4 \pi r_{\mathrm{vir}}^{3}} \frac{1}{g_{\mathrm{NFW}}(c)} \times \underbrace{\frac{1}{c x(1+c x)^{2}}}_{\equiv \tilde{\rho}_{\mathrm{NFW}}},
\end{aligned}
$$

with

$$
g_{\mathrm{NFW}}(c)=\frac{\log (1+c)-\frac{c}{1+c}}{c^{3}}
$$

provides a good fit to DM-only N-body simulations over a wide range of halo masses [66]. The associated gravitational potential is

$$
\tilde{\psi}_{\mathrm{NFW}}=\frac{\log (1+c x)}{c^{3} g_{\mathrm{NFW}}(c) x} .
$$


With $c=10$ and a scale radius of $a=20 \mathrm{kpc}$, the mass enclosed within $r_{\mathrm{vir}}=200 \mathrm{kpc}$ is $M_{\text {vir }}=9.9 \times 10^{11} M_{\odot}$. This falls within the broad range of values, $5 \times 10^{11} M_{\odot} \lesssim M_{\text {vir }} \lesssim$ $3 \times 10^{12} M_{\odot}$, that have been inferred for the Milky Way. Even though several studies have recently suggested that the Milky Way might be less massive and more concentrated than previously thought (see e.g. [67]), the values above are adequate for our purposes.

To model a dwarf Spheroidal (dSph) satellite of the Milky Way with an NFW profile, we note that observations are consistent with the known satellites having a mass of about $10^{7} M_{\odot}$ within their central $300 \mathrm{pc}$ [68], while a scale radius $a=0.62 \mathrm{kpc}$ fits the observed radial velocity dispersion of the stars [52]. The total mass of the dSph dark matter halos is difficult to determine, since their extent beyond the observed stellar distributions is largely unknown [69]. For definiteness, we will assume that these objects extend up to $r_{\text {vir }}=3 \mathrm{kpc}$, and contain a mass of $M_{\text {vir }}=1.3 \times 10^{8} M_{\odot}$, which is the value obtained by extrapolating the NFW halo with the parameters determined above from more robust observations of the central region. Hence, $c=4.8$ in eq. (3.8), which is substantially lower than the typical values $c \sim 20$ for halos of this size found in simulations [70].

Since

$$
\tilde{\psi}^{\max }=\tilde{\psi}(0)=\frac{1}{c^{2} g_{\mathrm{NFW}}(c)},
$$

the maximum velocity of any bound particle in an NFW halo is

$$
v_{\mathrm{NFW}}^{\max }=\sqrt{\frac{2}{c^{2} g_{\mathrm{NFW}}(c)} \frac{G M_{\mathrm{vir}}}{r_{\mathrm{vir}}}} .
$$

For the Galaxy, $v_{\mathrm{NFW}}^{\max } \approx 537.3 \mathrm{~km} / \mathrm{s}$, and for a typical dwarf, $v_{\mathrm{NFW}}^{\max } \approx 43.7 \mathrm{~km} / \mathrm{s}$.

\subsection{Einasto profile}

In addition to describing the luminosity profiles of early-type galaxies and bulges and the surface density of hot gas in clusters, the Einasto profile is as good a fit as the NFW profile, if not better, to simulated galaxy-sized dark matter halos:

$$
\begin{aligned}
\rho_{\text {Ein }} & =\rho_{0} \exp \left(-\frac{2}{\gamma}\left[\left(\frac{r}{a}\right)^{\gamma}-1\right]\right) \\
& =\frac{M_{\text {vir }}}{4 \pi r_{\text {vir }}^{3}} \frac{1}{g_{\operatorname{Ein}}(c)} \times \underbrace{\exp \left(-\frac{2}{\gamma}\left[(c x)^{\gamma}-1\right]\right)}_{\equiv \tilde{\rho}_{\text {Ein }}} .
\end{aligned}
$$

Here,

$$
g_{\operatorname{Ein}}(c)=\frac{\left(\frac{2}{\gamma}\right)^{-3 / \gamma} \exp (2 / \gamma)}{c^{3} \gamma}\left(\Gamma\left(\frac{3}{\gamma}\right)-\Gamma\left(\frac{3}{\gamma}, \frac{2 c^{\gamma}}{\gamma}\right)\right),
$$

where $\Gamma(x)$ and $\Gamma(s, x)$ are the usual gamma function and the incomplete gamma function respectively [71]. A value of $\gamma=0.17$ provides a good fit to galactic- and cluster-sized halos in N-body simulations [72, 73]. As with the NFW profile, we take $c=10$ and $r_{\text {vir }} \approx 200 \mathrm{kpc}$ for the Milky Way, which falls roughly along the concentration-mass relation for the WMAP5 cosmology [74]. 
The associated potential can be written as [71]:

$$
\begin{aligned}
\tilde{\psi}_{\text {Ein }}= & \frac{c}{\Gamma(3 / \gamma)-\Gamma\left(3 / \gamma, 2 c^{\gamma / \gamma}\right)} \\
& \times\left[\frac{\Gamma(3 / \gamma)-\Gamma\left(3 / \gamma, 2(c x)^{\gamma} / \gamma\right)}{c x}+\left(\frac{2}{\gamma}\right)^{1 / \gamma} \Gamma\left(2 / \gamma, 2(c x)^{\gamma} / \gamma\right)\right] .
\end{aligned}
$$

\subsection{Burkert profile}

The fact that the best NFW fit to the observations of dSphs can be far less concentrated than expected from simulations suggests that the NFW profile provides a poor fit to the dynamics of some dSphs. In particular, detections of distinct stellar sub-populations provide mass estimates at different radii for Fornax and Sculptor that are consistent with cored potentials, but largely incompatible with cusped profiles [75, 76].

The Burkert profile is a cored profile that appears to provide a good fit to the DM distribution in dSph galaxies [77]. Its density is given by

$$
\begin{aligned}
\rho_{\mathrm{Bur}} & =\frac{\rho_{0}}{\left(1+\frac{r}{a}\right)\left(1+\frac{r^{2}}{a^{2}}\right)} \\
& =\frac{M_{\mathrm{vir}}}{4 \pi r_{\mathrm{vir}}^{3}} \frac{1}{g_{\mathrm{Bur}}(c)} \times \underbrace{\frac{1}{(1+c x)\left(1+(c x)^{2}\right)}}_{\equiv \tilde{\rho}_{\mathrm{Bur}}},
\end{aligned}
$$

with

$$
g_{\text {Bur }}(c)=\frac{\log \left(1+c^{2}\right)+2 \log (1+c)-2 \arctan (c)}{4 c^{3}} .
$$

The gravitational potential generated by this profile is

$$
\tilde{\psi}_{\mathrm{Bur}}=\frac{\pi c x-2(1+c x) \arctan (c x)+2(1+c x) \log (1+c x)+(1-c x) \log \left(1+(c x)^{2}\right)}{4 c^{3} x g_{\mathrm{Bur}}(c)} .
$$

A scale radius of $a=650 \mathrm{pc}$ and $\rho_{0}=1.8 \times 10^{8} M_{\odot}$ in eq. (3.16) were found in [78] to fit the kinematics of Draco. With these parameters, and for $r_{\text {vir }}=3 \mathrm{kpc}$, we obtain $M_{\text {vir }}=6 \times 10^{8} M_{\odot}$, which is slightly larger than our NFW model of a dwarf. To make comparisons easier, we keep $c=4.6$, but we rescale down $\rho_{0}$ to match $M_{\text {vir }}=1.3 \times 10^{8} M_{\odot}$.

\subsection{Galactic bulge and disk}

In addition to the dark halo, the gravitational potential of the Milky Way receives contributions from stars in the disk and the bulge. Indeed, the baryonic contribution to the gravitational field is the dominant one in the central region of the Galaxy and will play a crucial role in determining the dark matter velocity distribution. We model the baryons by adding a central bulge and a disk. Following [79], we take a spherically-symmetric Hernquist potential for the bulge,

$$
\tilde{\psi}_{\text {bulge }}=\frac{M_{\text {bulge }}}{M_{\text {vir }}} \frac{1}{x+\frac{c_{0}}{r_{\text {vir }}}},
$$


where $c_{0} \sim 0.6 \mathrm{kpc}$ and $M_{\text {bulge }}=1.5 \times 10^{10} M_{\odot}$. We represent the disk by a spherical distribution that approximates the mass and circular velocity of the exponential disk:

$$
\tilde{\psi}_{\text {disk }}=\frac{M_{\text {disk }}}{M_{\text {vir }}} \frac{1-\exp \left(-\frac{r_{\text {vir }} x}{b_{\text {disk }}}\right)}{x},
$$

with $b_{\text {disk }} \sim 4 \mathrm{kpc}$ and $M_{\text {disk }}=5 \times 10^{10} M_{\odot}$. Although the galactic disk is certainly flattened, the distribution in eq. (3.20) contains the same amount of mass interior to $x$ as an exponential disk and matches its circular speed with error no more than $\sim 15 \%$ (c.f. fig. 2.17 in [60]). We discuss the effects of a flattened potential in section 6 .

\section{Phase-space distribution function}

As explained in section 2, the kinematics of the annihilating dark matter particles in the gravitational field of the halo can be fully described by the DF, $f$.

Most studies of DM indirect detection implicitly take $f$ to be the product of one of the density profiles discussed in Sec. 3 and a Maxwell-Boltzmann velocity distribution independent of the position in the halo. As discussed below, such a distribution function cannot in general describe a system of collisionless particles moving under the influence of a smooth gravitational potential. Indeed, only for the density in eq. (3.1) does a Maxwell-Boltzmann velocity distribution satisfy the Boltzmann equation. The results of numerical simulations and better observations have, for the most part, been incorporated in the spatial part of $f$, without updating the velocity distribution. For the most discussed neutralino models featuring a mostly s-wave velocity independent cross-section one can ignore this discussion, since the production rate in eq. (2.9) does not depend on the shape of the velocity distribution as long as it is properly normalized. This is no longer the case for the DM scenarios with Sommerfeld enhancement that have been recently discussed in connection with the observed cosmic-ray anomalies. A more practical reason for neglecting the velocity distribution is that it is much more difficult to measure than the density. The resolution of numerical simulations has increased dramatically in recent years, but the number of particles is still too small to sample the possible velocities at each point in the synthetic halo. To estimate the velocity distribution at the particular location of the solar system, an average over 100 randomly distributed sample spheres centered at $8.5 \mathrm{kpc}$ was performed in [80] to capture about $10^{4}$ particles among the billion particles in Via Lactea II, an N-body simulation of a Milky-Way-size galaxy. These results provide insight into the non-gaussian shape of the velocity distribution and on the influence of substructure, but an extension to map the inner and outer parts of the halo is clearly unfeasible. An empirical fit to the local distribution was found in [81] from a suite of cosmological simulations, and a full phase-space distribution consistent with a fit to the observed circular rotation of the Galaxy was considered in [82].

Such studies of the local distribution are motivated by the conflicting results of direct detection experiments, which are sensitive to the velocity of the DM particles even for swave annihilating DM. We here extend the study of the velocity distribution to the rest of the halo, as required in studies of DM indirect detection, by using the formalism developed by Eddington. Although, we leave the consideration of a velocity anisotropy or a fit to numerical simulations for future work [83], our method captures the effects of deviations from gaussianity found in studies of the local neighborhood, while maintaining a self-consistent phase-space distribution that satisfies the Boltzmann equation. 
For a spherical system confined by a known gravitational potential $\psi$, we can find a unique ergodic (i.e. isotropic in velocity space) distribution function using Eddington's result [60]. In this case, $f$ is a function of the energy per unit mass and can be written as

$$
f(\tilde{\epsilon})=\frac{1}{8 \sqrt{2} \pi^{3} \sqrt{G^{3} r_{\mathrm{vir}}^{3} M_{\mathrm{vir}}}} \frac{1}{g(c)} \times \tilde{f}(\tilde{\epsilon}),
$$

where

$$
\tilde{f}(\tilde{\epsilon}) \equiv \int_{0}^{\tilde{\epsilon}} \frac{\mathrm{d} \tilde{\psi}}{\sqrt{\tilde{\epsilon}-\tilde{\psi}}} \frac{\mathrm{d}^{2} \tilde{\rho}}{\mathrm{d} \tilde{\psi}^{2}} .
$$

It is not possible in general to invert $\tilde{\psi}(x)$ in order to express $\tilde{\rho}$ as a function of $\tilde{\psi}$. We can, nevertheless, express the derivative in eq. (4.2) as

$$
\frac{\mathrm{d}^{2} \tilde{\rho}}{\mathrm{d} \tilde{\psi}^{2}}=\left(\frac{\mathrm{d} \tilde{\psi}}{\mathrm{d} x}\right)^{-2}\left(\frac{\mathrm{d}^{2} \tilde{\rho}}{\mathrm{d} x^{2}}-\left(\frac{\mathrm{d} \tilde{\psi}}{\mathrm{d} x}\right)^{-1} \frac{\mathrm{d}^{2} \tilde{\psi}}{\mathrm{d} x^{2}} \frac{\mathrm{d} \tilde{\rho}}{\mathrm{d} x}\right) .
$$

Then eq. (4.2) reads:

$$
\tilde{f}(\tilde{\epsilon})=\int_{0}^{\tilde{\epsilon}} \frac{\mathrm{d} \tilde{\psi}}{\sqrt{\tilde{\epsilon}-\tilde{\psi}}}\left(\frac{\mathrm{d} \tilde{\psi}}{\mathrm{d} x^{\prime}}\right)^{-2}\left(\frac{\mathrm{d}^{2} \tilde{\rho}}{\mathrm{d} x^{\prime 2}}-\left(\frac{\mathrm{d} \tilde{\psi}}{\mathrm{d} x}\right)^{-1} \frac{\mathrm{d}^{2} \tilde{\psi}}{\mathrm{d} x^{\prime 2}} \frac{\mathrm{d} \tilde{\rho}}{\mathrm{d} x^{\prime}}\right)
$$

where the integrand and the limits of integration are a function of the position $x$. We numerically solve $\tilde{\psi}=\tilde{\psi}(x)$ for $x$ when performing the integration in eq. (4.4) to find $\tilde{f}(\tilde{\epsilon})$. We show in figs. 1 and 2 the distribution function for the Galaxy and for a classical dSph.

The three-dimensional velocity distribution can be written as

$$
\begin{aligned}
P_{\boldsymbol{x}}(\boldsymbol{v}) \mathrm{d}^{3} \boldsymbol{v} & =\frac{1}{\sqrt{8} \pi^{2}} \sqrt{\left(\frac{r_{\mathrm{vir}}}{G M_{\mathrm{vir}}}\right)^{3}} \frac{\tilde{f}\left(\psi-v^{2} / 2\right)}{\tilde{\rho}(\boldsymbol{x})} \mathrm{d}^{3} \boldsymbol{v} \\
& =\frac{1}{\sqrt{8} \pi^{2}} \frac{\tilde{f}\left(\tilde{\psi}-\tilde{v}^{2} / 2\right)}{\tilde{\rho}(\boldsymbol{x})} \mathrm{d}^{3} \tilde{\boldsymbol{v}},
\end{aligned}
$$

which is a function of $v^{2}$ for an isotropic spherical system, $P_{\boldsymbol{x}}(\boldsymbol{v})=P_{\boldsymbol{x}}\left(v^{2}\right)$, and $\tilde{v}^{2} \equiv \frac{r_{\mathrm{vir}}}{G M_{\mathrm{vir}}} v^{2}$.

One can obtain the one-dimensional distribution of the magnitude of the velocity by performing the angular integration in $\boldsymbol{v}$-space:

$$
\begin{aligned}
P_{r}\left(v^{2}\right) \mathrm{d} v & =\frac{\sqrt{2}}{\pi} \sqrt{\left(\frac{r_{\mathrm{vir}}}{G M_{\mathrm{vir}}}\right)^{3}} v^{2} \frac{\tilde{f}}{\tilde{\rho}} \mathrm{d} v \\
& =\frac{\sqrt{2}}{\pi} \tilde{v}^{2} \frac{\tilde{f}}{\tilde{\rho}} \mathrm{d} \tilde{v} .
\end{aligned}
$$

This distribution function is normalized as follows:

$$
\int_{0}^{\infty} P_{r}\left(v^{2}\right) \mathrm{d} v=1
$$

where the upper integration limit can be replaced by the maximum velocity, $\sqrt{2 \psi(r)}$. 
Figs. 3, 4, 5, 6, and 7 show the distribution function in eq. (4.6). At large distances, the distribution can be well-approximated by a Maxwell-Boltzmann shape. However, as we move towards the center of the halo, large departures from gaussianity are evident. In addition, as we move closer to the center of the halo, the actual distribution function shifts to lower values of the velocity, in stark contrast with the uniform Maxwell-Boltzmann (MB) shape with constant velocity dispersion, which is usually assumed in studies of indirect detection of dark matter.

In the Galaxy, the added gravitational potential of the disk and the bulge contributes to alleviate the cooling of dark matter particles towards the center. Although this improves the agreement with a description based on a constant MB distribution at moderate distances, large departures from gaussianity are still evident within the central $1 \mathrm{kpc}$.

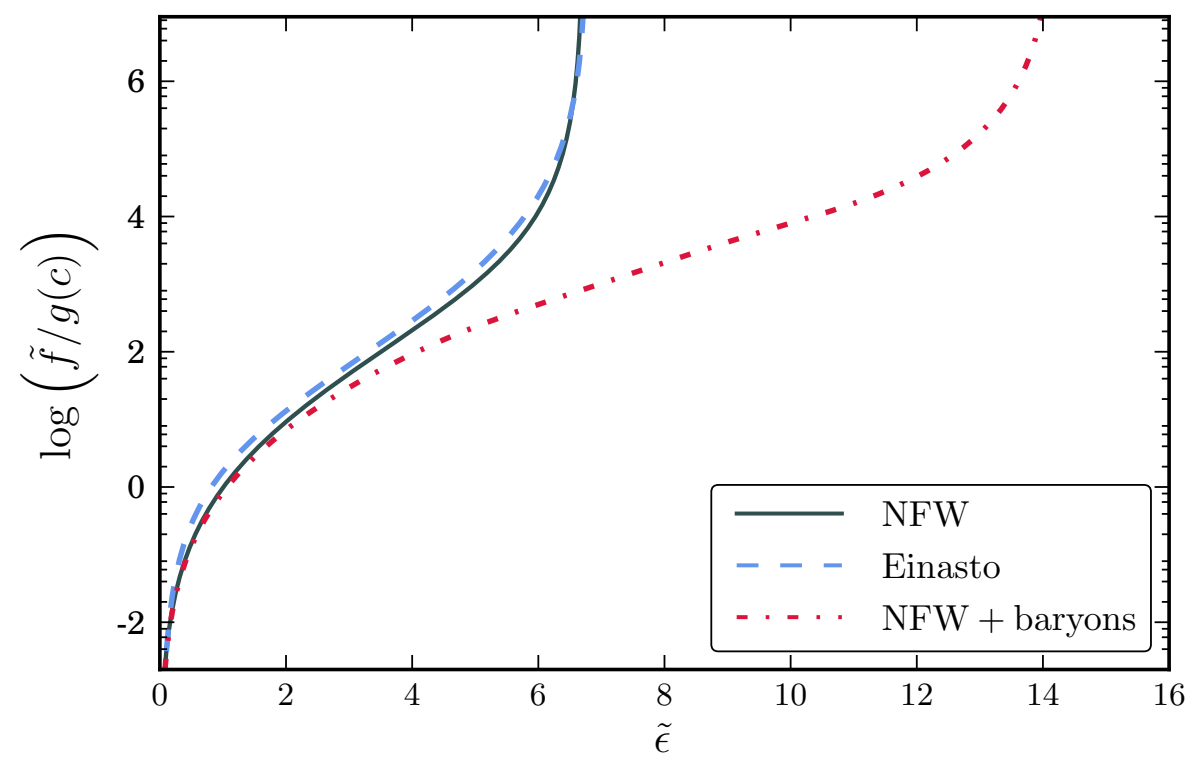

Figure 1. Phase-space distribution function of the dark matter in the Galaxy, eq. (4.4), assuming an NFW profile, with and without a baryonic disk, or a dark matter only Einasto profile.

\subsection{The relative velocity distribution}

The yield of SM particles from dark matter annihilation depends on the distribution of relative velocities at a given location, as shown in section 2 . For a spherical systems with an ergodic distribution function, $\tilde{f}=\tilde{f}\left(v^{2}\right)$ at any point in the halo. Then, the individual velocity distributions in eq. (2.8) do not depend on the six components of the velocities, but only on the combinations:

$$
P_{\boldsymbol{x}}\left(v_{1,2}^{2}\right)=P_{\boldsymbol{x}}\left(v_{\mathrm{cm}}^{2}+v_{\mathrm{rel}}^{2} / 4 \pm \boldsymbol{v}_{\mathrm{cm}} \cdot \boldsymbol{v}_{\mathrm{rel}}\right) .
$$

Using spherical coordinates in $\boldsymbol{v}_{\text {rel }}$-space, with the $z$-axis in the direction of the relative velocity vector,

$$
\begin{array}{r}
P_{\boldsymbol{x}, \text { rel }}\left(v_{\mathrm{rel}}\right) \equiv 8 \pi^{2} v_{\mathrm{rel}}^{2} \int_{0}^{\infty} \mathrm{d} v_{\mathrm{cm}} v_{\mathrm{cm}}^{2} \int_{-1}^{1} \mathrm{~d} z P_{\boldsymbol{x}}\left(v_{\mathrm{cm}}^{2}+v_{\mathrm{rel}}^{2} / 4+v_{\mathrm{cm}} v_{\mathrm{rel}} z\right) \times \\
P_{\boldsymbol{x}}\left(v_{\mathrm{cm}}^{2}+v_{\mathrm{rel}}^{2} / 4-v_{\mathrm{cm}} v_{\mathrm{rel}} z\right)
\end{array}
$$




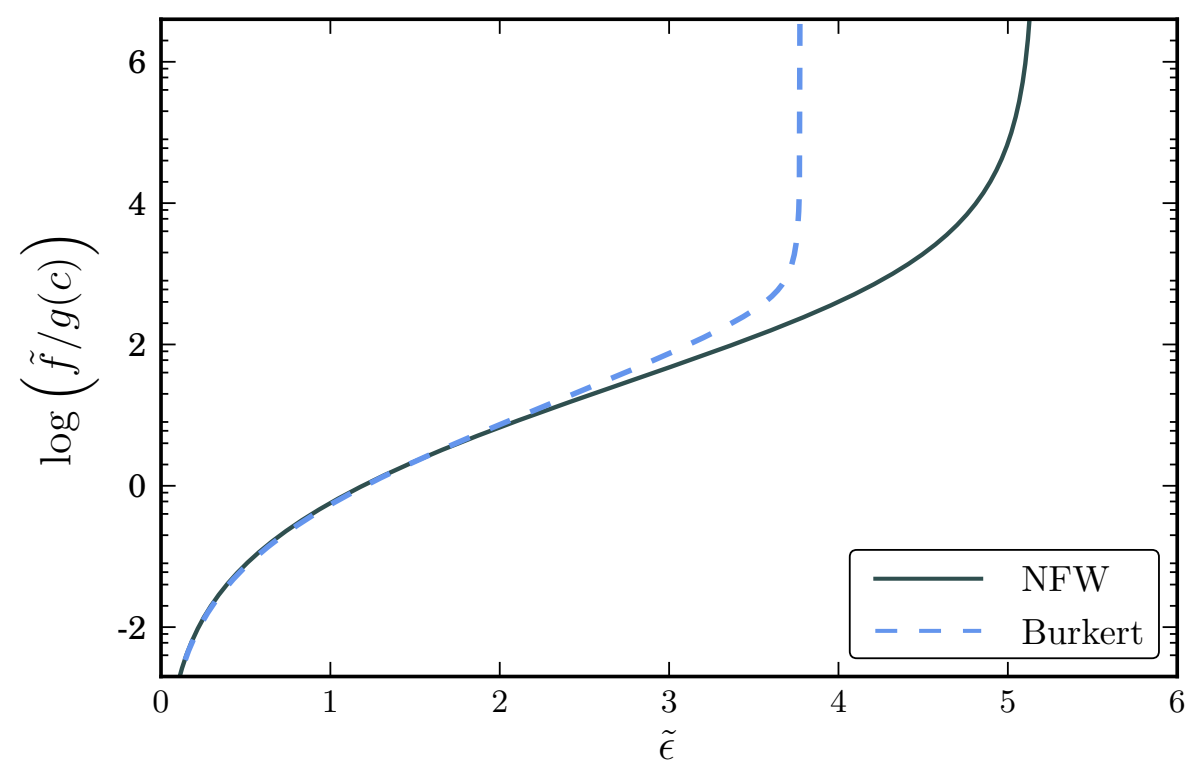

Figure 2. Phase-space distribution function of the dark matter in a dSph with an NFW or a cored Burkert profile.

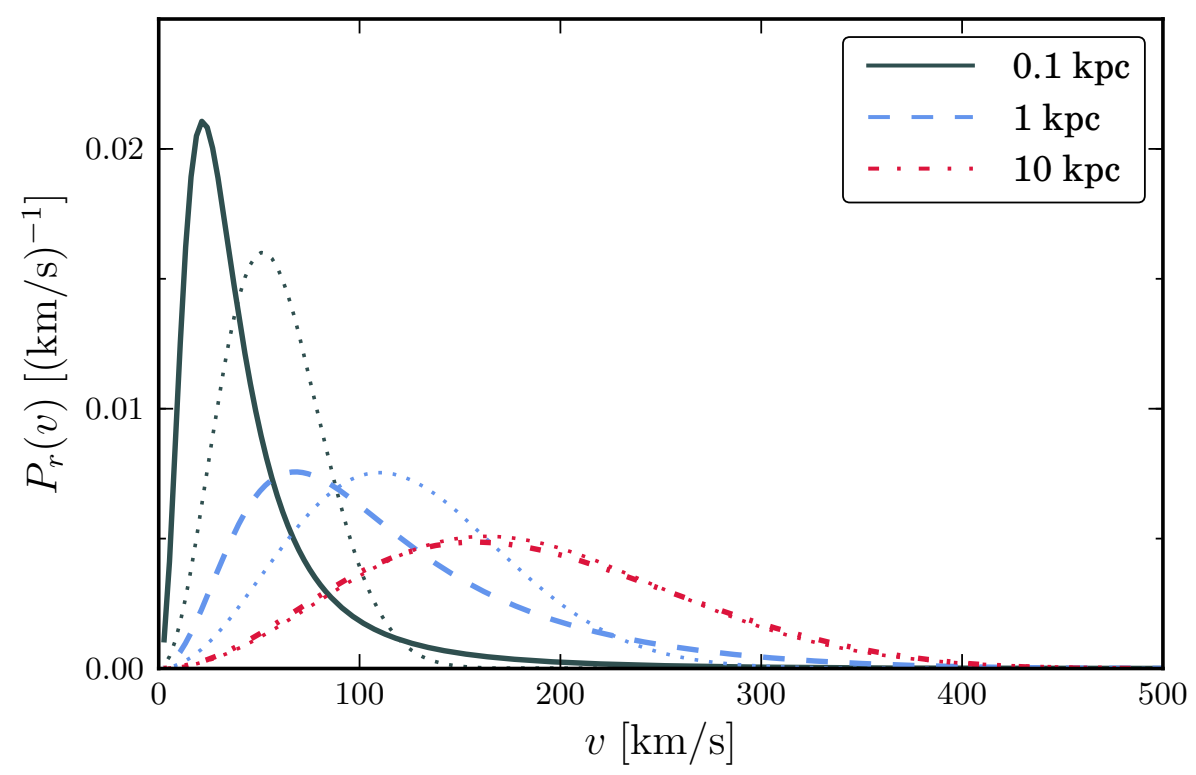

Figure 3. Velocity distribution, eq. (4.6), for the an NFW galaxy without baryons. The dotted lines show a Maxwell-Boltzmann distribution with the same velocity dispersion.

where $z$ is the cosine of the angle between the relative and the center of mass velocities. In appendix A we write the expression above explicitly in terms of the individual phase-space distribution function. 


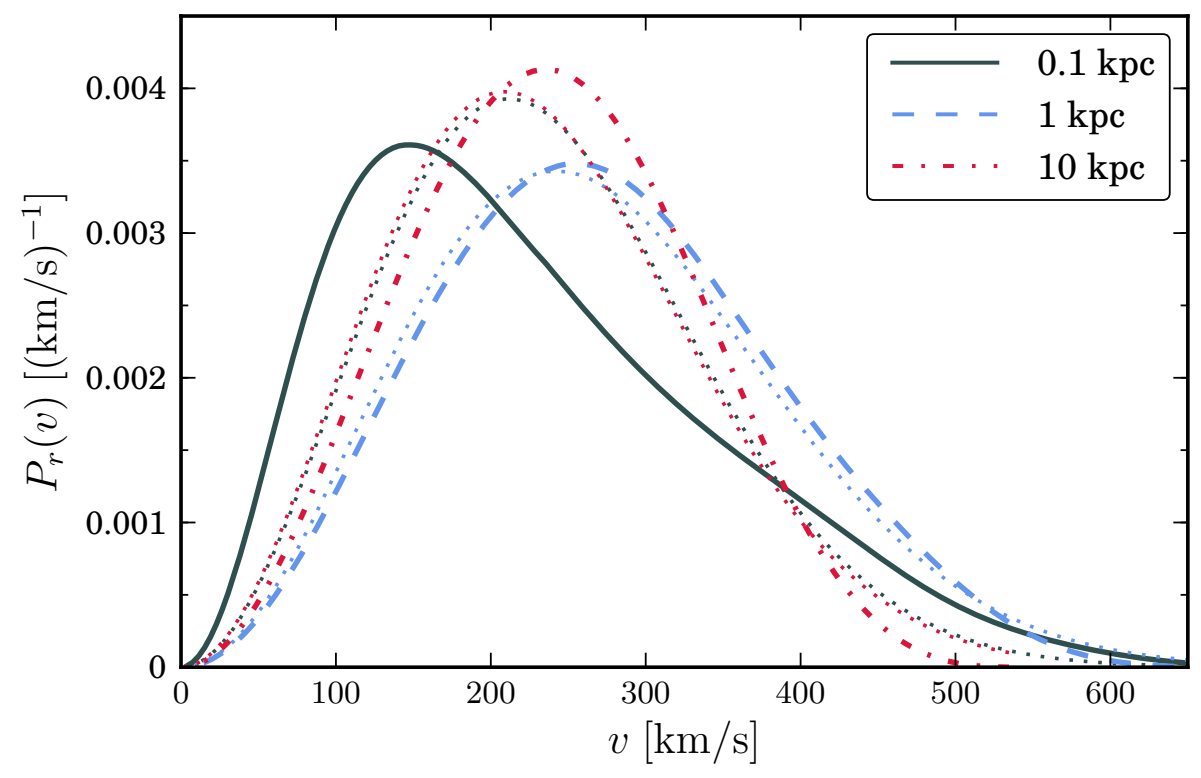

Figure 4. Velocity distribution, eq. (4.6), for an NFW galaxy with disk and bulge eqs. (3.20,3.19).

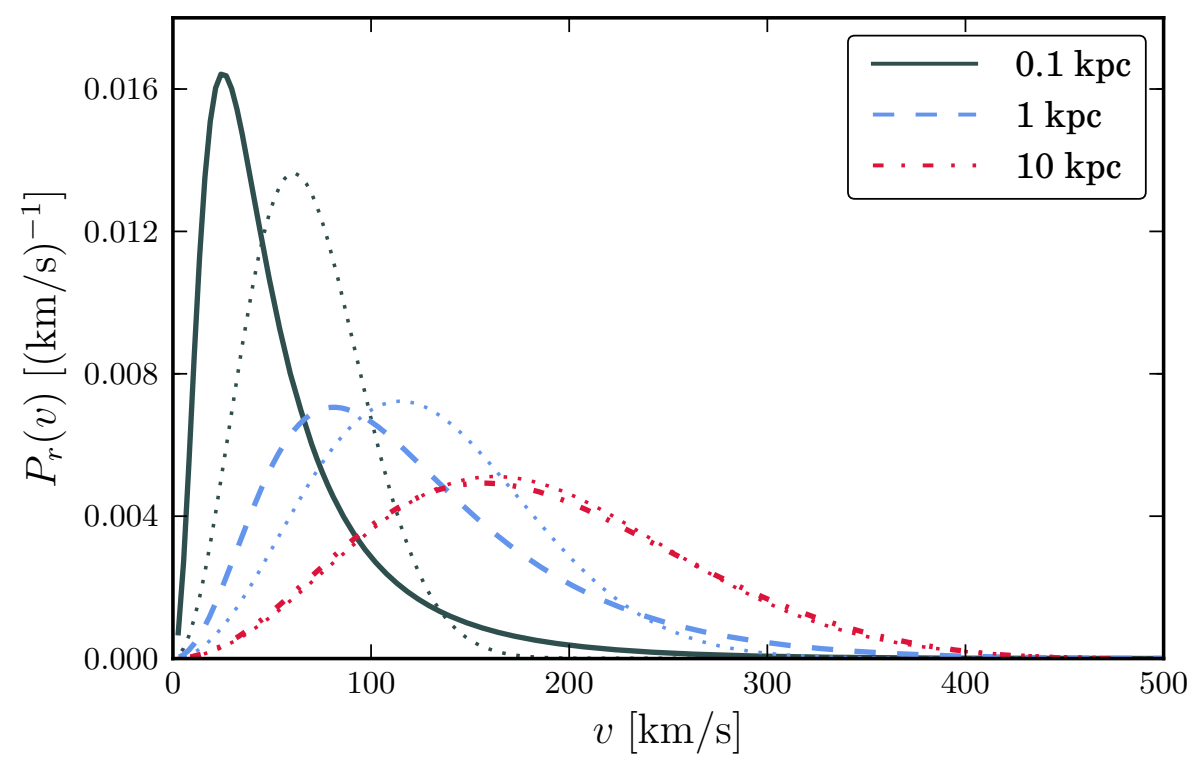

Figure 5. Velocity distribution, eq. (4.6), for an Einasto galaxy-sized dark matter halo.

Results for a Maxwell-Boltzmann distribution We can use the formalism developed above to recover the well-known results of the Standard Halo [61], which models the galaxy after a singular isothermal sphere profile. 


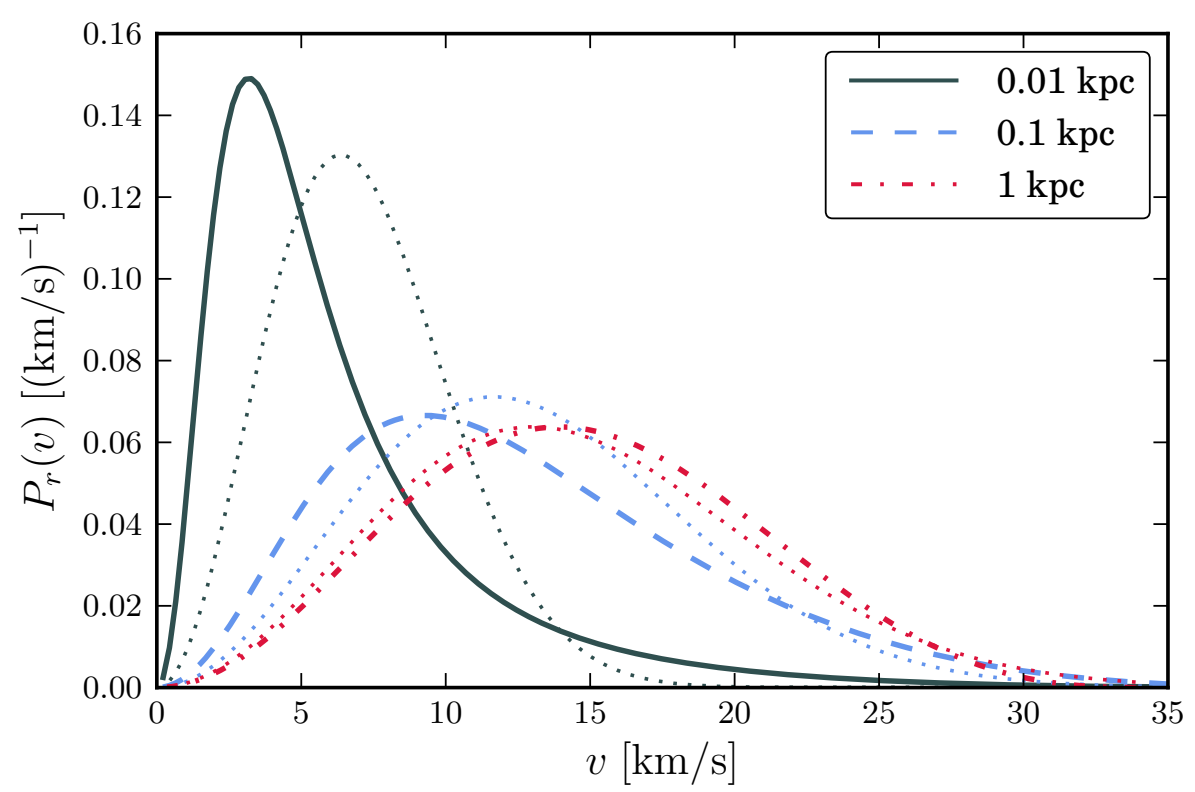

Figure 6. Velocity distribution, eq. (4.6), for a classical dSph satellite of the Milky Way with an NFW profile.

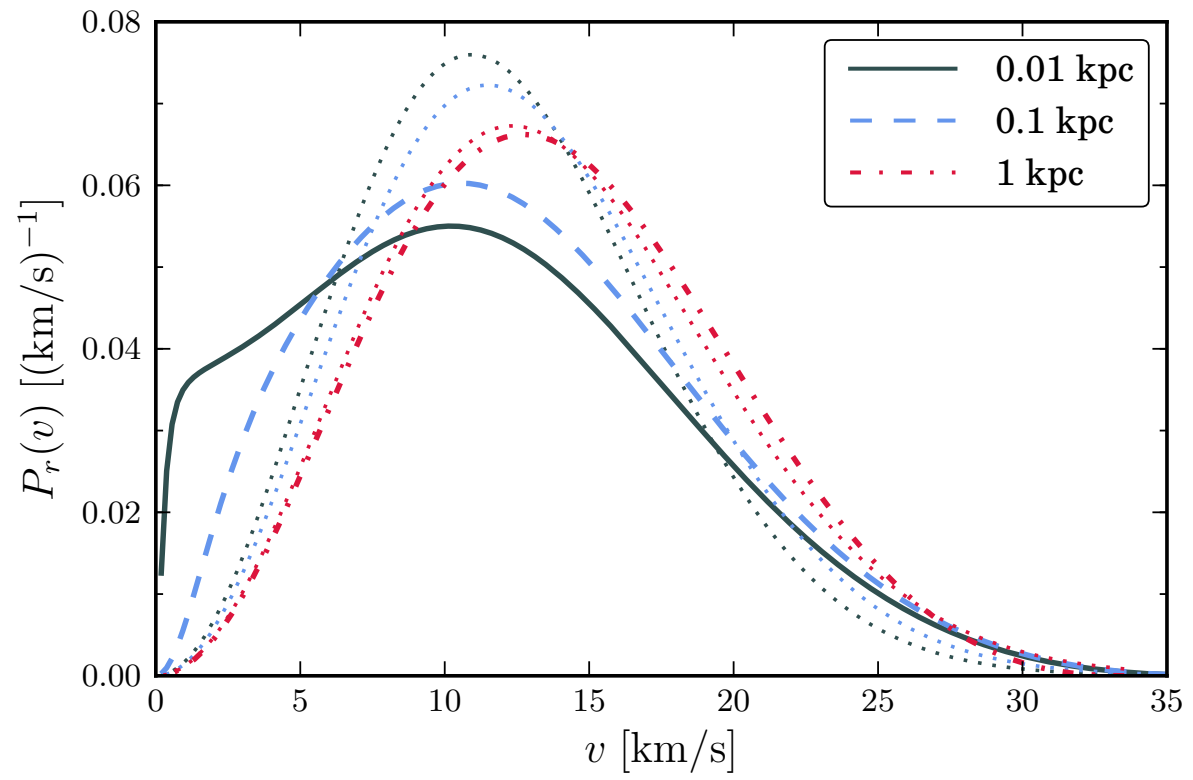

Figure 7. Velocity distribution, eq. (4.6), for a classical dSph satellite of the Milky Way with a more appropriate Burkert profile.

The phase-space distribution function that gives rise to the density in eq. (3.1) is [60]

$$
f(\epsilon)=\frac{\rho_{1}}{\left(2 \pi \sigma^{2}\right)^{3 / 2}} \exp \left(\frac{\psi-\frac{v^{2}}{2}}{\sigma^{2}}\right),
$$


which results in a velocity distribution of the Maxwell-Boltzmann type:

$$
P_{\boldsymbol{x}}^{M B}(\boldsymbol{v})=\frac{1}{\left(2 \pi \sigma^{2}\right)^{3 / 2}} \exp \left(\frac{-v^{2}}{2 \sigma^{2}}\right) .
$$

Note that the MB velocity distribution does not depend on the spatial coordinates, i.e. $\sigma$ is a constant.

A straightforward application of eq. (4.9) gives the relative velocity distribution:

$$
P_{\text {rel }}^{M B}\left(v_{\text {rel }}\right)=4 \pi v_{\text {rel }}^{2} \frac{1}{\left(2 \pi\left(2 \sigma^{2}\right)\right)^{3 / 2}} \exp \left(\frac{-v_{\text {rel }}^{2}}{2\left(2 \sigma^{2}\right)}\right) .
$$

It is a particularity of the MB distribution that the relative velocity distribution also has the same functional form, i.e. it is another MB distribution. The one-dimensional relative velocity dispersion is, of course, doubled in the center of mass with respect to that of the individual particles.

The velocity dispersion $\sigma$ can be related to observable quantities, such as the mass interior to radius $r$ or the circular speed. Alternatively, it can be determined from N-body simulations, as was done in [84], where a constant MB distribution was used to estimate the Sommerfeld correction to Via Lactea II, an N-body simulation of a Milky-Way-size galaxy.

Relative velocity distribution for an NFW or Burkert profile Figs. 8, 9, and 10 show the distribution function for the relative velocity in eq. (4.9). As expected, the velocity dispersion changes with the distance to the center of the halo, and the distribution can be highly non-gaussian. For an NFW density profile, a Maxwell-Boltzmann distribution function is a good approximation to the exact relative velocity distribution for distances of the order of the scale radius ( $\sim 20 \mathrm{kpc}$ for the Galaxy and $\sim 0.15 \mathrm{kpc}$ for a $\mathrm{dSph})$ and larger. This is reasonable, since the density profile behaves like $\propto 1 / r^{2}$ in this region, similar to the SIS.

Adding a baryonic disk and bulge to the Galaxy increases the gravitational potential, which raises the relative velocity dispersion. Also, the Maxwell-Boltzmann distribution is a better fit for distances above $\sim 1 \mathrm{kpc}$. However, as we enter the innermost region, the departures from gaussianity are evident again.

\subsection{Consistency with Jeans analysis}

The distribution function contains all the information about the behaviour of the DM particles in phase-space. A limited understanding of the dependence of the velocity distribution on the position of the halo can be obtained in a more direct way by taking velocity moments of the collisionless Boltzmann equation. In this manner, one obtains Jeans equation that can be used to compute the one-dimensional velocity dispersion.

For a spherical system with an isotropic velocity distribution, the Jeans equation reads [60]

$$
\begin{aligned}
\bar{v}_{r}^{2}(r) & =\frac{1}{\rho(r)} \int_{r}^{\infty} \mathrm{d} r^{\prime} \frac{\mathrm{d} \Phi}{\mathrm{d} r^{\prime}} \rho\left(r^{\prime}\right) \\
& =\frac{G M_{\text {vir }}}{r_{\text {vir }}} \frac{1}{\tilde{\rho}(x)} \int_{\infty}^{x} \mathrm{~d} x^{\prime} \frac{\mathrm{d} \tilde{\psi}}{\mathrm{d} x^{\prime}} \tilde{\rho}\left(x^{\prime}\right),
\end{aligned}
$$

which, for an NFW halo, can be expressed analytically in terms of elementary and polylogarithm funcions. 


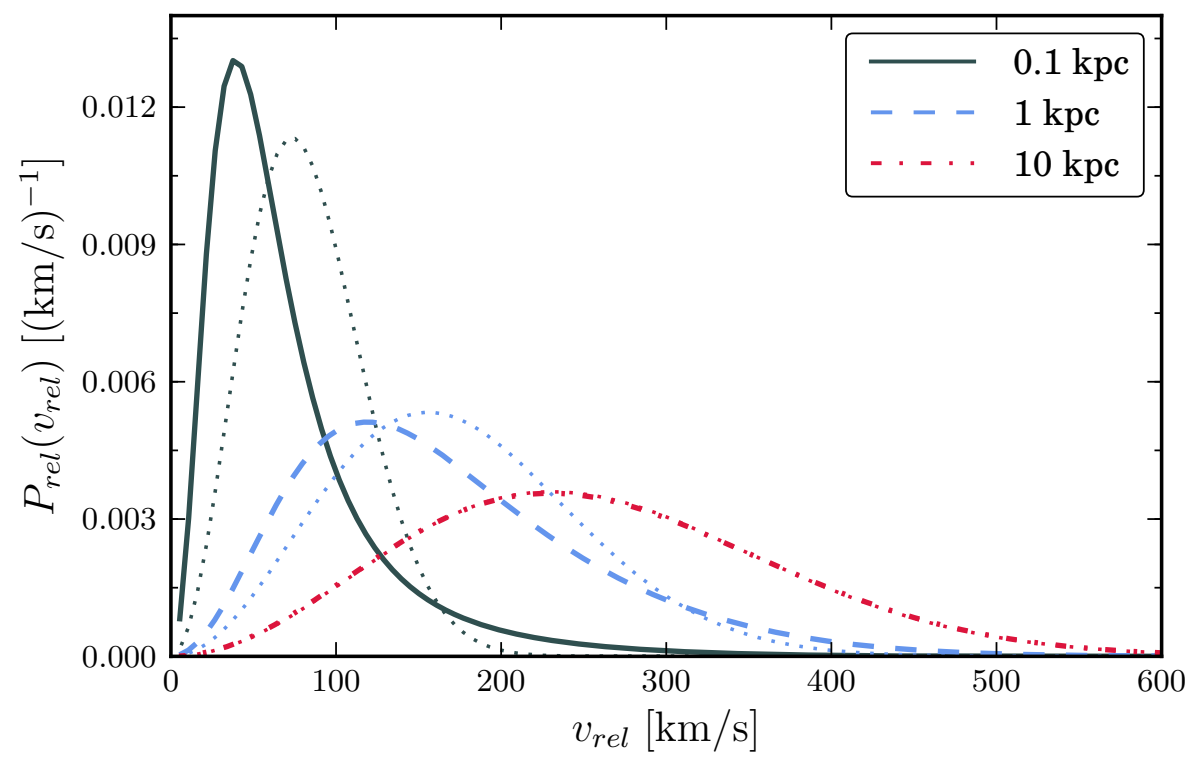

Figure 8. Relative velocity distribution, eq. (4.9), for an NFW dark matter halo only. The dotted lines show a Maxwell-Boltzmann distribution with the same velocity dispersion: $\sigma=$ $51.8 \mathrm{~km} / \mathrm{s}, 110.1 \mathrm{~km} / \mathrm{s}, 163.2 \mathrm{~km} / \mathrm{s}$ for $r=0.1 \mathrm{kpc}, 1 \mathrm{kpc}, 10 \mathrm{kpc}$.

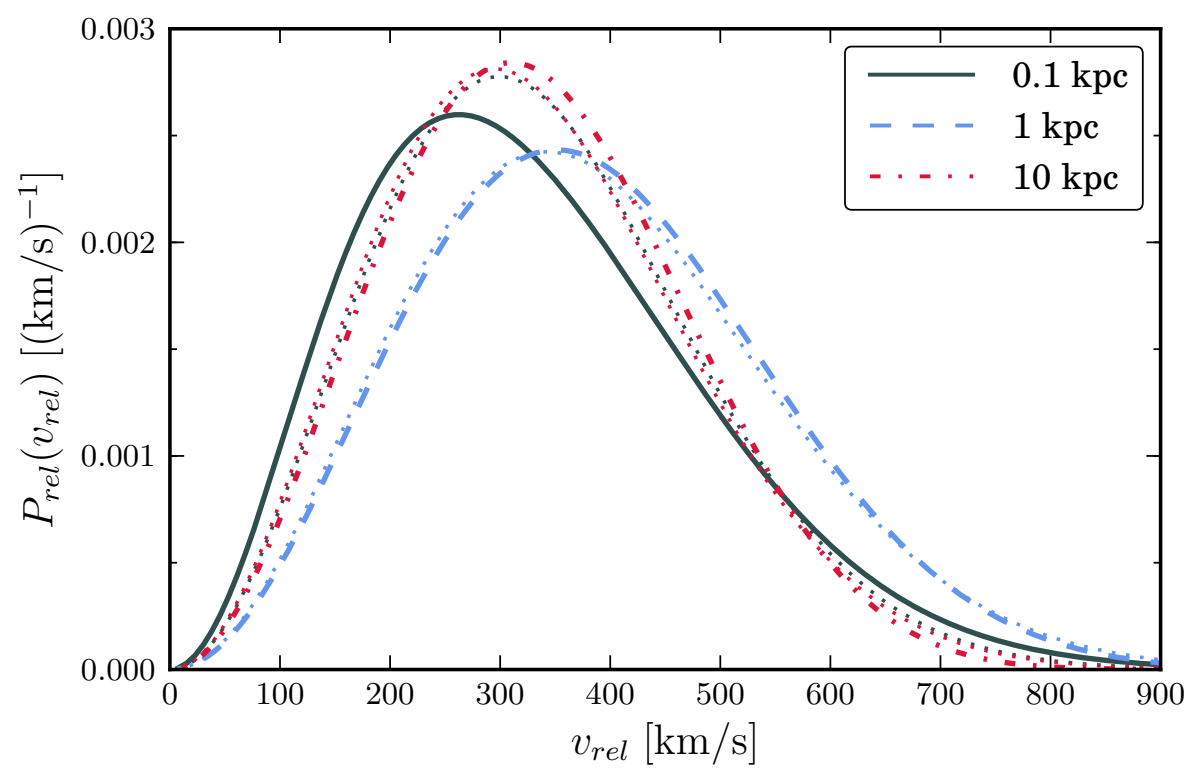

Figure 9. Relative velocity distribution, eq. (4.9), for an NFW Galaxy with disk and bulge. The dotted lines show a Maxwell-Boltzmann distribution with the same velocity dispersion: $\sigma=$ $211.4 \mathrm{~km} / \mathrm{s}, 242.2 \mathrm{~km} / \mathrm{s}, 208.8 \mathrm{~km} / \mathrm{s}$ for $r=0.1 \mathrm{kpc}, 1 \mathrm{kpc}, 10 \mathrm{kpc}$.

On the other hand, we can find the dispersion directly from the distribution function 


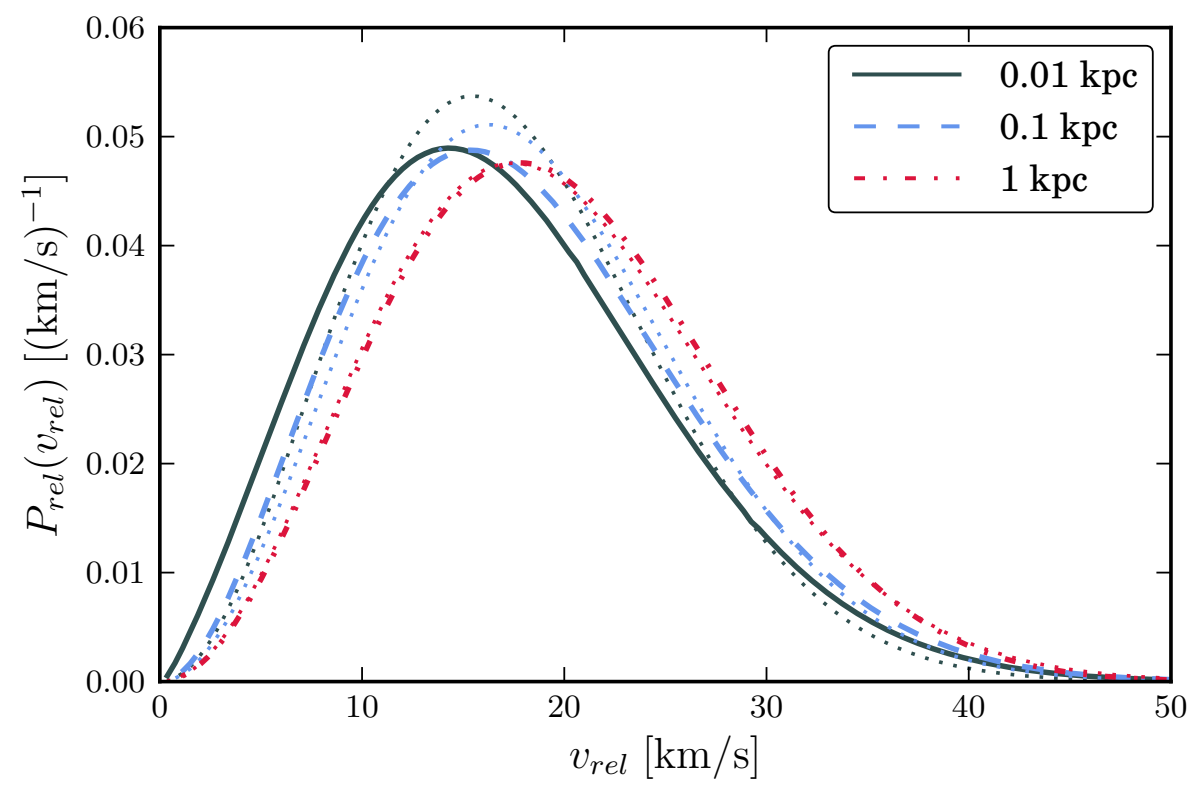

Figure 10. Relative velocity distribution, eq. (4.9), for a classical dSph satellite of the Milky Way with a Burkert profile. The dotted lines show a Maxwell-Boltzmann distribution with the same velocity dispersion: $\sigma=10.9 \mathrm{~km} / \mathrm{s}, 11.5 \mathrm{~km} / \mathrm{s}, 12.3 \mathrm{~km} / \mathrm{s}$ for $r=0.01 \mathrm{kpc}, 0.1 \mathrm{kpc}, 1 \mathrm{kpc}$.

in eq. (4.6):

$$
3 \bar{v}_{r}^{2}(r)=\int_{0}^{\sqrt{2 \tilde{\psi}}} \mathrm{d} v v^{2} P_{r}(v) .
$$

To test our numerical procedure, we check that the results of eq. (4.13) and eq. (4.14) agree within numerical accuracy.

Since the one-particle distribution is not Maxwellian, we cannot in principle use eq. (4.12) to find the dispersion in the relative velocity. However, as fig. (11) shows, the approximation $\bar{v}_{r e l}^{2}=2 \bar{v}_{1}^{2}$ holds well for a wide range of distances. This behaviour is consistent with Jeans type analyses to higher orders that show how the lowest order moment hardly constrains the form of the potential [85]. As a consequence, for dark matter particles annihilating via a p-wave process (or, more trivially, s-wave), we can get accurate fluxes simply with a Jeans analysis. This was the strategy adopted in [55], where it was also pointed out that nongaussianities at small galactocentric distances cannot be captured with this formalism. This is particularly important for dark matter particles whose annihilation in the halo is enhanced by the Sommerfeld effect. Since we have at our disposal the full relative velocity distribution, we are able to evaluate accurate fluxes for dark matter annihilations with an arbitrary velocity dependence.

\section{$5 \quad$ Fluxes from dark matter annihilations}

With the results of the previous section we can evaluate the production rate eq. (2.9) for an arbitrary velocity dependent annihilation cross-section. In the following, we study gamma-ray fluxes from the galactic center or a dSph satellite and synchrotron emission produced by $e^{ \pm}$in 


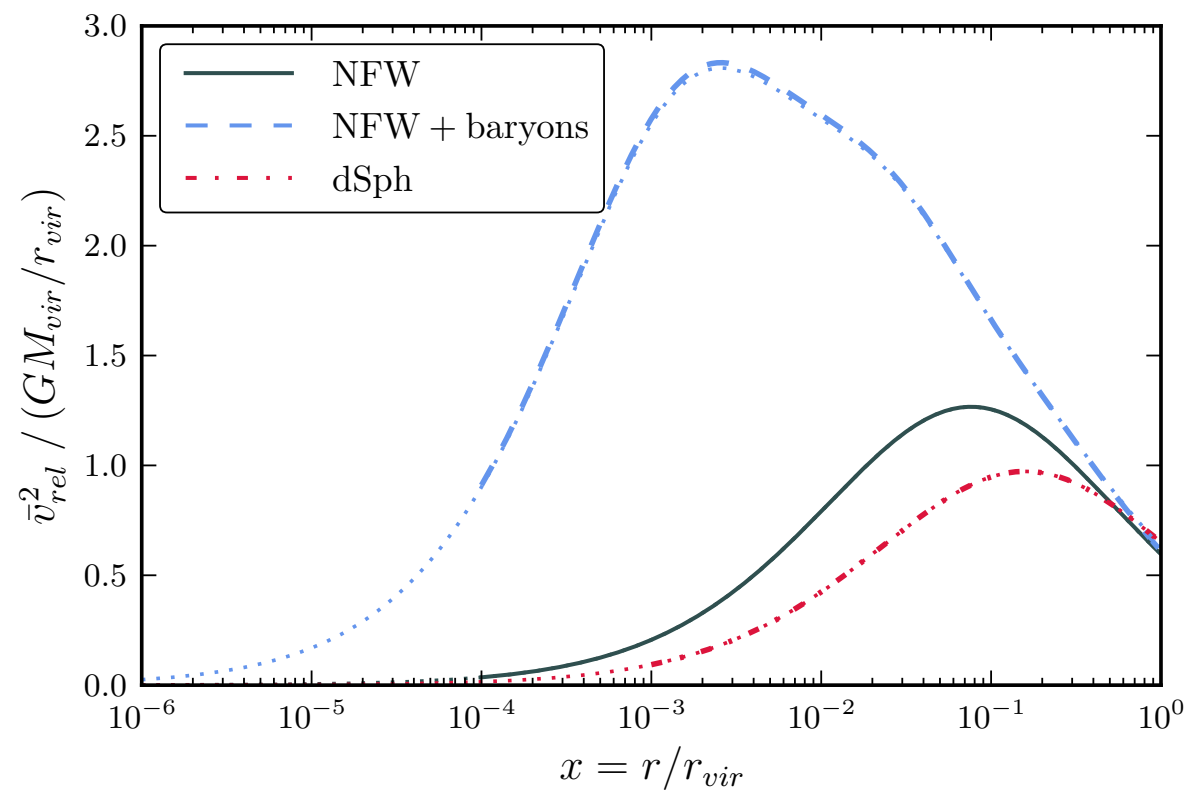

Figure 11. One dimensional relative velocity dispersion. The thick lines show the result of using the exact velocity distribution, while the thinner dotted lines use a Jeans analysis and assume that the one-particle distribution is $\mathrm{MB}$, like in eq. (4.12). Note that in physical units, the line corresponding to the dSph would be down by a factor of $M_{\text {vir }}^{d S p h} r_{\text {vir }}^{g a l} / M_{\text {vir }}^{g a l} r_{\text {vir }}^{d S p h} \sim 1.5 \times 10^{3}$

the galactic magnetic field. We are motivated by the possibility that the recent observations of the rising positron fraction at $\mathrm{GeV}$ energies could be due to $\mathrm{DM}$ annihilations. For this explanation to be viable, DM must dominantly annihilate into leptons with a larger-thanthermal cross-section. If so, there ought to be significant photon fluxes associated with these leptons: either directly generated (via bremsstrahlung of charged particles or the decay of $\pi^{0}$ ) or the synchrotron radiation generated by the leptons in the central galactic magnetic field. Such fluxes provide some of the most stringent constraints for these scenarios [48], since the large annihilation cross-section is compounded with the increasing DM density in the center of the halo. In addition, the velocity dependence of the annihilation cross-section enhances the emission from the center as pointed out in [55] in the context of an approximate Jeans analysis. We here quantify this effect using the formalism developed above.

Let us start considering the photon fluxes. To simplify the discussion, suppose we are interested in photons of any energy and that the cross-section does not depend on energy. Then we have

$$
\frac{\mathrm{d} \Gamma}{\mathrm{d} V}=\frac{N_{\gamma}}{m_{\chi}^{2}}\left\langle\sigma v_{\mathrm{rel}}\right\rangle \rho_{\chi}^{2},
$$

where $N_{\gamma}$ is the number of photons that ultimately result from each annihilation, and we take $B R_{i}=1$. The differential flux along a direction specified in general by two angles, $\psi$ and $\phi$, is

$$
\mathrm{d} \Phi_{\gamma}=\mathrm{d} l \frac{N_{\gamma}}{4 \pi m_{\chi}^{2}}\left\langle\sigma v_{\text {rel }}\right\rangle \rho_{\chi}^{2}
$$

To find the total flux, we must integrate along the line-of-sight $l$ in all directions contained 
by the solid angle $\Delta \Omega$. Any spatially-varying quantities in equation eq. (5.2), i.e. quantities that are functions of $\boldsymbol{r}=\boldsymbol{r}(l, \psi, \phi)$, must be taken into account in this integration. The mass and photon yield per annihilation do not depend on location, and the density certainly does, but what about the interaction rate $\langle\sigma v\rangle^{4}$ ? Integrating 5.2, the total flux from the galactic center is[2]

$$
\Phi_{\gamma}(\Delta \Omega)=\frac{N_{\gamma}\langle\sigma v\rangle_{\mathrm{MB}}}{4 \pi m_{\chi}^{2}} \bar{J}(\Delta \Omega) \Delta \Omega
$$

where $\langle\sigma v\rangle_{\mathrm{MB}}$ is the interaction rate with the usual spatially-independent boost and the so-called " $J$-factor" is

$$
J(\psi)=\int \mathrm{d} l \frac{\langle\sigma v\rangle(l)}{\langle\sigma v\rangle_{\mathrm{MB}}} \rho_{\chi}^{2}(l),
$$

with the bar designating an average over the solid angle $\Delta \Omega$. We chose the $z$-axis to point in the direction of the center of the halo, and we have assumed cylindrical symmetry. If we take the velocity distribution as Maxwell-Boltzmann and spatially constant then $\langle\sigma v\rangle(l)=\langle\sigma v\rangle_{\mathrm{MB}}$ everywhere and

$$
J(\psi) \rightarrow J_{\mathrm{MB}}(\psi)=\int \mathrm{d} l \rho_{\chi}^{2}(l)
$$

This is the usual calculation.

In general, though, $\langle\sigma v\rangle$ is location-dependent and must be kept inside the volume integral. We work with the multiplicative change in the flux from using the Eddington equation or Jeans equation instead of Maxwell-Boltzmann:

$$
\mathcal{F} \equiv J / J_{\mathrm{MB}}=\Phi_{\gamma} / \Phi_{\gamma, \mathrm{MB}}
$$

DarkSUSY [86] code rewritten in Python was used to calculate this quantity, given a profile model and Sommerfeld model.

We are also interested on the emission of synchrotron and inverse Compton (IC) radiation in the galactic center. To find the synchrotron luminosity in the galaxy $L_{\nu}$, consider the energy distribution of electrons $[2,87]$

$$
\frac{\mathrm{d} n_{e}}{\mathrm{~d} E}=\frac{\mathrm{d} \Gamma}{\mathrm{d} V} \frac{Y_{e}(>E)}{P_{e}(E)}
$$

where $\mathrm{d} \Gamma / \mathrm{d} V$ is the local $\mathrm{DM}$ annihilation rate, $Y_{e}(>E)$ is the number of electrons and positrons created by each annihilation, and

$$
P_{e}(E)=\frac{2 e^{4} B^{2} E^{2}}{3 m_{e}^{4} c^{7}}
$$

is the electron power spectrum or energy loss rate.

The power radiated (as photons) at frequency $\nu$ by an electron with energy $E$ at galactocentric radius $r$ is

$$
P_{\gamma}(\nu, E)=\frac{\sqrt{3} e^{3}}{m_{e} c^{2}} B(r) F\left(\frac{\nu}{\nu_{c}(E)}\right),
$$

where $K_{n}$ is the modified Bessel function of order $n$ and

$$
F\left(\frac{\nu}{\nu_{c}(E)}\right)=\frac{\nu}{\nu_{c}(E)} \int_{0}^{\infty} \mathrm{d} y K_{5 / 3}(y) .
$$

\footnotetext{
${ }^{4}$ From now on we suppress the subscript "rel"
} 
Integrating over the electron energy $E$ and over the volume, we obtain the luminosity

$$
L_{\nu}=\int \mathrm{d} V \int_{m_{e}}^{m_{\chi}} \mathrm{d} E \frac{\mathrm{d} n_{e}}{\mathrm{~d} E} P_{\gamma}(\nu, E) \text {, }
$$

which becomes

$$
L_{\nu}=\frac{\sqrt{3} e^{3}}{m_{e} c^{2}} \int \mathrm{d} V \frac{\mathrm{d} \Gamma}{\mathrm{d} V} B(r) \int_{m_{e}}^{m_{\chi}} \mathrm{d} E \frac{Y_{e}(>E)}{P_{e}(E)} F\left(\frac{\nu}{\nu_{c}(E)}\right) .
$$

We focus on the spatially-dependent part of the integrand. The function $P(E)$ is proportional to $B^{2}$, so we have

$$
L_{\nu} \sim \frac{4 \pi}{m_{\chi}^{2}} \int \mathrm{d} r r^{2}\langle\sigma v\rangle \rho_{\chi}^{2} B^{3} .
$$

Most previous work takes the quantity $\langle\sigma v\rangle$ as spatially constant, removing it from the integrand. To get an idea of the consequences of relaxing this approximation, we take a simple step-function as the model for the magnetic field. The relevant quantity is then

$$
L_{\nu} \sim \int_{0}^{r_{B}} \mathrm{~d} r r^{2}\langle\sigma v\rangle \rho_{\chi}^{2}
$$

where $r_{B}=1 \mathrm{kpc}$ is the radius at which we take the magnetic field as zero.

The multiplicative enhancement analogue to eq. (5.4) is then:

$$
\Gamma_{\mathrm{ann}}=4 \pi \int_{0}^{r} \mathrm{~d} r^{\prime} r^{\prime 2} \frac{\langle\sigma v\rangle\left(r^{\prime}\right)}{\langle\sigma v\rangle_{0}} \rho_{\chi}^{2}\left(r^{\prime}\right)
$$

where $r=r_{B}$ sets the size of the spherical volume where most of the emission is generated. The same expression can be used to estimate the change in the IC emission from the bulge, where the up-scattered starlight and IR photons is most plentiful [88]. Analogous to eq. (5.6), we focus on the quantity

$$
\mathcal{G} \equiv=\Gamma_{\text {ann }} / \Gamma_{\text {ann }, \mathrm{MB}}=L_{\nu} / L_{\nu, \mathrm{MB}}
$$

Given a DM density profile and a particle physics model defining $\sigma v$, we can calculate eqs. (5.6) and (5.16). We choose the density profiles in Sec. 3, and consider DM particles for which the cross-section times the flux is a decreasing function of $v_{\text {rel }}$.

\subsection{Sommerfeld enhancement}

The presence of a light force carrier mediating long range forces between the dark matter particles causes a singular behaviour of the Feynman amplitude, which invalidates the partialwave expansion $\sigma v_{\text {rel }}=a+b v_{\text {rel }}^{2}+\ldots$. This is the basis of the non-perturbative Sommerfeld enhancement [43-46]. Although an accurate calculation of the enhancement requires the resummation of processes with multiple exchanges of the mediator [89], an analytic solution can be found by approximating the Yukawa potential by the Hulthén potential [90-92]. The multiplicative change to the cross-section, called the Sommerfeld factor, is then

$$
S(v)=\frac{\pi \alpha_{\chi}}{v} \frac{\sinh \left(\frac{12 v}{\pi \xi}\right)}{\cosh \left(\frac{12 v}{\pi \xi}\right)-\cos \left(2 \pi \sqrt{\frac{6 \alpha_{\chi}}{\pi^{2} \xi}-\left(\frac{6 v}{\pi^{2} \xi}\right)^{2}}\right)}
$$


Here $\alpha_{\chi}$ is the coupling constant and $\xi$ is the ratio between the carrier mass and DM particle mass. This analytic expression is an excellent approximation to the numerical calculation. It accurately reproduces the $\sigma v \sim 1 / v$ behaviour that saturates to a constant $\sim 1 / v_{\text {min }}$, with $v_{\text {min }} \approx \xi$, and also captures the resonant behaviour, $\sigma v \sim 1 / v^{2}$, for particular values of $\xi$ [92]. The enhancement is then

$$
\frac{\langle\sigma(v) v\rangle(v)}{\langle\sigma v\rangle_{0}}=\int_{0}^{\infty} \mathrm{d} v S(v) P_{\text {rel }}(v),
$$

where $\langle\sigma v\rangle_{0}$ is the annihilation rate before invoking the Sommerfeld enhancement.

To illustrate our calculations, we consider the Arkani-Hamed et al. model [44], with $\alpha_{\chi}=10^{-2}$ and $\xi$ between $1.2 \times 10^{-3}$ and $2 \times 10^{-3}$. Within this range we find resonant behaviour, as well as the standard $1 / v$ regime that saturates at small velocities.

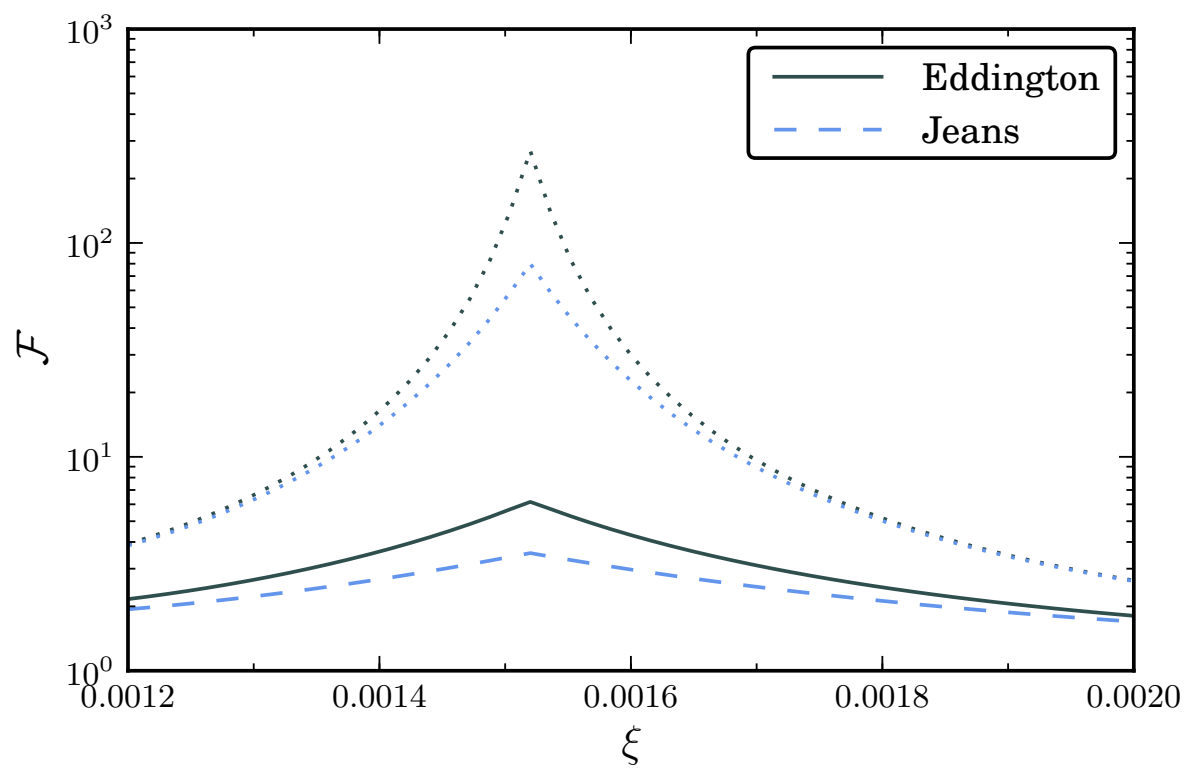

Figure 12. $\mathcal{F}$ calculated for the galactic center using a NFW profile with (solid) and without (dotted) baryonic components.

\section{Results and discussion}

Calculating the relative velocity distribution, eq. (4.9), involves a four-dimensional integration, which must be convolved according to eq. (5.18) before performing the volume integration in eqs. (5.6) and (5.16). To avoid this numerically challenging route, we generate a library of relative velocity distributions for each halo profile. We pick 350 (250) log-spaced locations between $r_{\text {vir }} \leq r \leq 10^{-4} r_{\text {vir }}\left(r_{\text {vir }} \leq r \leq 10^{-3} r_{\text {vir }}\right)$ in the Galaxy (dSph). At each location we sample $P_{\boldsymbol{r} \text {,rel }}$ for $10^{3}$ velocities between 0 and the escape velocity at the distance $r$ from the center ${ }^{5}$. We use these samples to evaluate eq. (5.18).

\footnotetext{
${ }^{5}$ The collection of velocity distributions can be downloaded from http://www . physics . wustl. edu/ferrer/ eddington/ .
} 


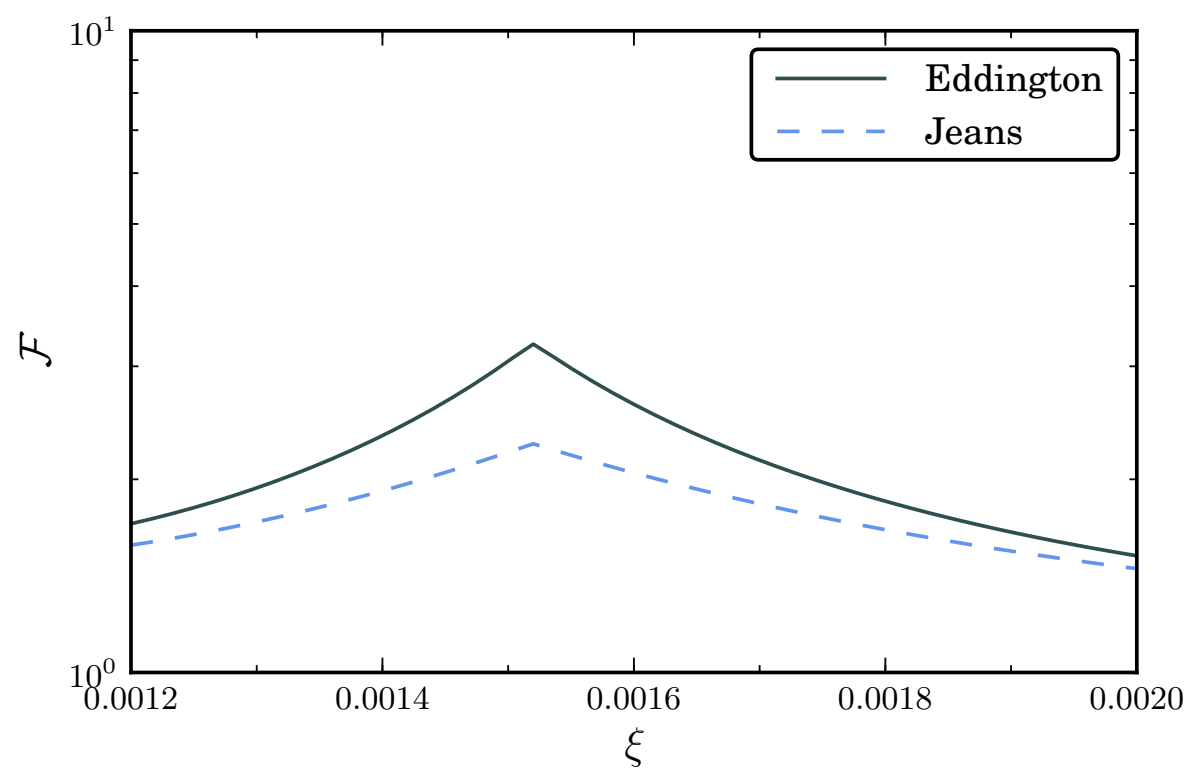

Figure 13. $\mathcal{F}$ calculated for the galactic center using an Einasto profile with baryonic components.

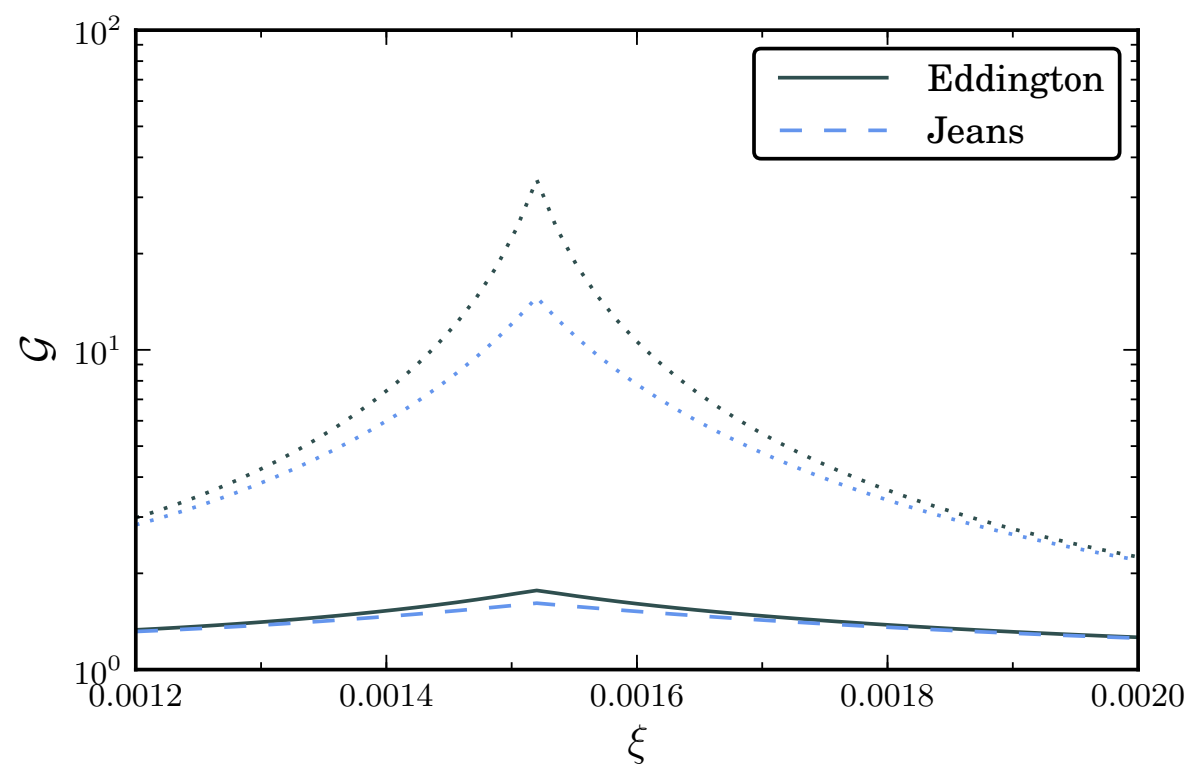

Figure 14. $\mathcal{G}$ calculated using a NFW profile with (solid) and without (dotted) baryonic components.

Figures 12 and 13 show the change in flux $\mathcal{F}$, eq. (5.6), for the galactic center, modeled with a NFW halo and Einasto halo, respectively. A solid angle of $10^{-5}$ sr is used, corresponding to the typical angular resolution of the Large Area Telescope on board of the Fermi satellite [93]. Two different calculations of $\mathcal{F}$ are plotted: that of a Maxwell-Boltzmann distribution with variable dispersion found from the Jeans equation [55], and our method 


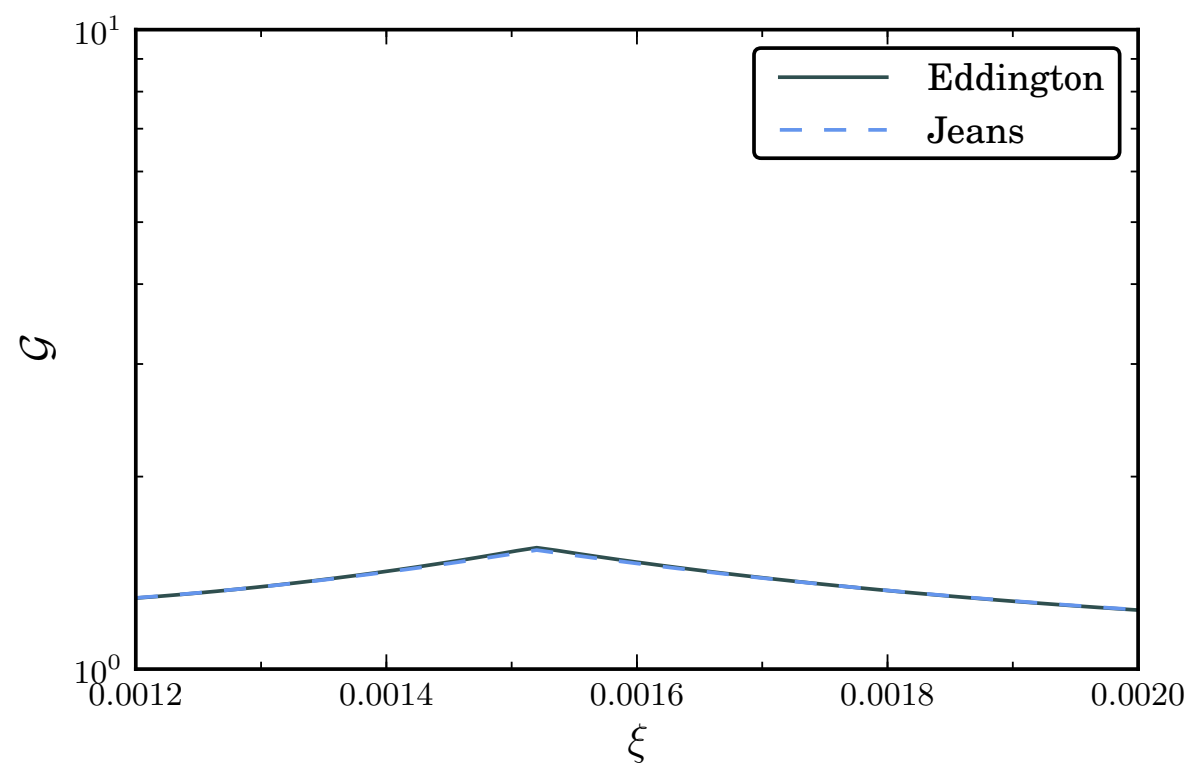

Figure 15. $\mathcal{G}$ calculated using an Einasto profile with baryonic components.

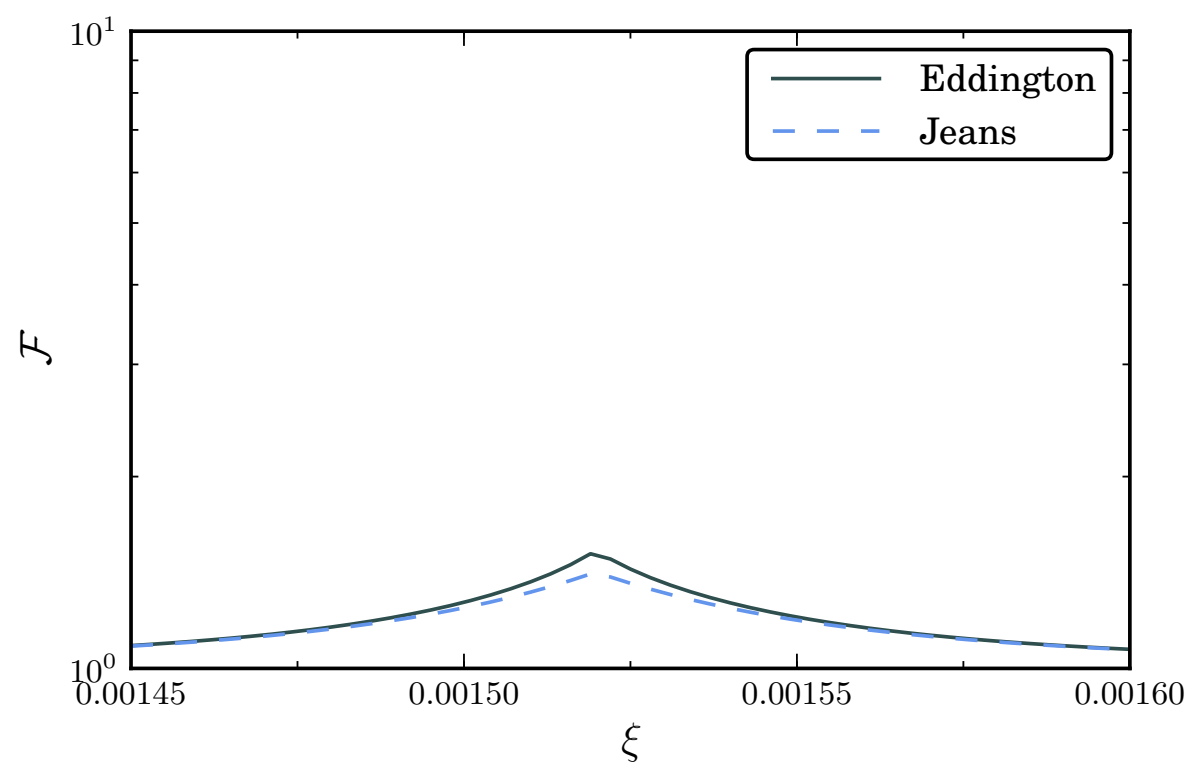

Figure 16. $\mathcal{F}$ calculated for the Draco dwarf spheroidal using a Burkert profile.

based on Eddington's equation. Some observations are of note. Using the Eddington equation generally grants predictions of stronger signals. This is because the distribution peak is at a lower velocity than in a Maxwell-Boltzmann distribution. With any distribution, enhancements are generally lower when baryonic components are taken into account, which 


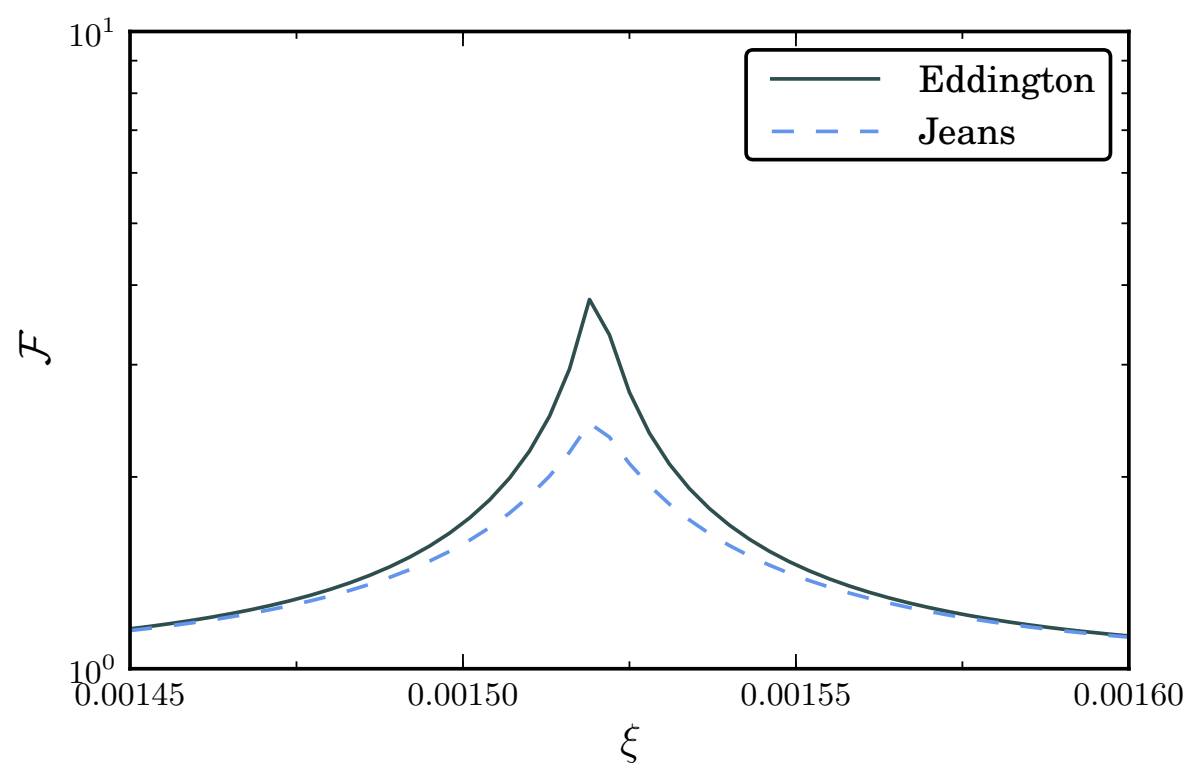

Figure 17. $\mathcal{F}$ calculated for the Draco dwarf spheroidal using a NFW profile.

\begin{tabular}{|c||c|c|c|c|c|c|}
\hline \multicolumn{1}{|c||}{$\xi / 10^{-3}$} & \multicolumn{3}{c|}{$\mathcal{F}_{\text {DF }} / \mathcal{F}_{\text {Jeans }}$} & \multicolumn{3}{c|}{$\mathcal{F}_{\text {DF }}$} \\
\hline & HO-NFW & NFW & Einasto & HO-NFW & NFW & Einasto \\
\hline 1.2 & 1.02 & 1.12 & 1.08 & 3.92 & 2.16 & 1.71 \\
1.3 & 1.05 & 1.20 & 1.13 & 6.63 & 2.66 & 1.94 \\
1.45 & 1.38 & 1.47 & 1.29 & 34.7 & 4.39 & 2.64 \\
1.51 & 2.70 & 1.70 & 1.41 & 178. & 5.87 & 3.16 \\
1.6 & 1.32 & 1.45 & 1.28 & 30.0 & 4.31 & 2.62 \\
1.8 & 1.03 & 1.16 & 1.11 & 5.17 & 2.45 & 1.85 \\
2.0 & 1.01 & 1.06 & 1.05 & 2.64 & 1.81 & 1.52 \\
\hline
\end{tabular}

Table 1. J-factor boosts for the galactic center. "HO" means halo-only; the other columns include baryonic components.

\begin{tabular}{|c||c|c|c|c|}
\hline \multicolumn{1}{|c||}{$\xi / 10^{-3}$} & \multicolumn{2}{c|}{$\mathcal{F}_{\mathrm{DF}} / \mathcal{F}_{\text {Jeans }}$} & \multicolumn{2}{c|}{$\mathcal{F}_{\mathrm{DF}}$} \\
\hline & Burkert & NFW & Burkert & NFW \\
\hline 1.48 & 1.01 & 1.02 & 1.16 & 1.33 \\
1.5 & 1.02 & 1.08 & 1.27 & 1.69 \\
1.52 & 1.08 & 1.64 & 1.53 & 4.07 \\
1.54 & 1.02 & 1.08 & 1.27 & 1.68 \\
1.56 & 1.01 & 1.02 & 1.16 & 1.33 \\
\hline
\end{tabular}

Table 2. $J$-factor boosts for the Draco dwarf spheroidal.

is obviously due to the extra mass that increases the velocity of the DM particles ${ }^{6}$ and it

${ }^{6}$ When the cross-section increases with velocity, as in $p$-wave annihilation, baryons increase the flux. 
is also important to notice that the addition of these baryonic models lessen the difference between the Jeans analysis predictions and the Eddington predictions - significant deviation from a Maxwell-Boltzmann distribution occurs at a smaller radius (compare figures 8 and 9). However, since much of the volume contributing to the line-of-sight observation is at the center, this deviation is still important.

Table 5.1 shows some specific values of $\mathcal{F}$. Our Eddington based calculation shows that when the variation in the velocity distribution of the DM particles is taken into account, gamma-ray fluxes are a factor of $\sim 1-10$ larger than expected from the standard estimate. When this effect is estimated using the Jeans equation [55] we typically recover fluxes within $\sim 20 \%$ of the more accurate Eddington prediction. In some particular cases, e.g. when the Sommerfeld enhancement is resonant, the exact prediction can be more than 100 times larger than the usual rough estimate, and about three times larger than what a Jeans analysis would bear.

Figures 16 and 17 plot $\mathcal{F}$ for Draco, taken as an exemplary dwarf spheroidal, modeled using a Burkert and NFW profile, respectively. No baryonic components are included, since they play a sub-dominant role in the dynamics of these galaxies. Because of the distance, the solid angle consumes the entire object (it is nearly a point-source), so the entire volume contributes to the signal. Since, as we have seen, a Maxwell-Boltzmann shaped distribution is accurate except at small radii, the flux enhancement is mostly due to the variation of the dispersion and not the shape of the distribution, a deviation being significant only for a small range of Sommerfeld models. Table 5.1 gives some possible values of $\mathcal{F}$.

Figures 14 and 15 plot $\mathcal{G}$ centered on the galaxy. The increase borne by our calculation is similar to that for dwarf spheroidals, $\sim 20-50 \%$ since, again, the signal comes primarily from larger radii.

By this point it is prudent to stress that these calculations are dependent on the "cutoff radius", at and below which the DM density is taken to be constant. DarkSUSY uses a default value of $10^{-5} \mathrm{kpc}$, which is much smaller than the typical resolution of numerical N-body simulations. We take a much more conservative approach, keeping both density and velocity distribution constant below $10^{-4} r_{\text {vir }}=2 \times 10^{-2} \mathrm{kpc}\left(10^{-3} r_{\mathrm{vir}}=3 \times 10^{-3} \mathrm{kpc}\right)$ for the Galaxy (Draco). The velocity distribution, in any case, becomes more and more non-Maxwellian at smaller and smaller radii. In particular, if the same calculation took enhancements calculated from distributions down to $10^{-5} r_{\text {vir }}$ or further, the impact of the spatial dependence of the velocity distribution would be much more pronounced, and our estimates are conservative in this respect.

Our calculation is self-consistent in the sense that our distribution function correctly describes DM as a collisionless system confined by a known gravitational potential. At the same time, our modelling of the galactic is necessarily simplified and does not include all the components for a complete description.

For instance, we have not allowed for any anisotropy in the velocity distribution. The anisotropy is expected to be small close to the center and increase in the outer region [94]. Since most of the annihilations occur close to the center, isotropy seems a fair assumption. However, the DF at a given position of the halo is sensitive to all orbits with energy greater than the gravitational potential and is affected, even in the central region, by the outer velocity anisotropy [95]. Indeed, [81] finds the Eddington calculation to be an inferior fit to some galaxy-scale simulations. These and other simulations (see, e.g. [96]) also suggest some anisotropy in the halo, which we plan to consider in future studies.

Also, we have focused on the smooth part of the halo, but small-scale structure is seen 
in N-body simulations. Substructure was shown in [97] to weaken somewhat the constraints from the inner galaxy. This is so because cold subhalos are more likely to survive in the outer galaxy and dominate the local signal, but they would have been tidally disrupted in the central regions. The presence of a dark disk with low dispersion velocity, suggested by simulations that include baryons, also seems to boost the local emission [98]. However, the dependence of the velocity distribution with the location in the halo was not taken into account in these studies, and there are doubts about the presence of significant substructure in the local vicinity [42].

Non-spherical baryonic disk A limitation of our analysis is that it assumes that the system is spherical. We have chosen a potential for the disk, following [79], that mimics the gravitational pull of a more realistic flattened system with an accuracy of $\sim 10 \%$. Even though Eddington's work has been extended to axisymmetric distributions [99], the formalism is far from trivial. Nevertheless, we can estimate the effects of a flattened disk by means of a simpler Jeans analysis, which does not need the full phase-space distribution function. The Jeans equation, eq. (4.13), may also be expressed in cylindrical coordinates $(R, z)$ :

$$
\begin{aligned}
& \bar{v}_{R}^{2}(R, z)=\bar{v}_{z}^{2}(R, z)=\frac{1}{\rho_{\mathrm{DM}}(R, z)} \int_{z}^{\infty} \mathrm{d} z \rho_{\mathrm{DM}}\left(R, z^{\prime}\right) \frac{\partial \Phi_{\mathrm{tot}}}{\partial z^{\prime}}, \\
& \bar{v}_{\phi}^{2}(R, z)=\bar{v}_{R}^{2}+\frac{R}{\rho_{\mathrm{DM}}} \frac{\partial\left(\rho_{\mathrm{DM}} \bar{v}_{R}^{2}(R, z)\right)}{\partial R}+R \frac{\partial \Phi_{\mathrm{tot}}}{\partial R} .
\end{aligned}
$$

Here, $\Phi_{\text {tot }}$ is the total potential, with contributions from the dark matter and the baryons.

To check the validity of the spherical disk model, we use eq. (4.13) to calculate $\bar{v}^{2}$, and compare it to the analogous calculation, using eq. (6.1), with the more realistic cylindrical disk model [60]

$$
\rho_{\mathrm{d}}(R, z)=\Sigma_{\mathrm{d}} e^{-R / R_{\mathrm{d}}}\left(\frac{\alpha_{0}}{2 z_{0}} e^{-|z| / z_{0}}+\frac{\alpha_{1}}{2 z_{1}} e^{-|z| / z_{1}}\right),
$$

taking $R_{d}=4 \mathrm{kpc}, z_{0}=0.3 \mathrm{kpc}$, and $z_{1}=1 \mathrm{kpc}$. The potential is

$$
\Phi_{\mathrm{d}}(R, z)=-\frac{4 G \Sigma_{\mathrm{d}}}{R_{\mathrm{d}}} \int_{-\infty}^{\infty} \mathrm{d} z^{\prime} \zeta\left(z^{\prime}\right) \int_{0}^{\infty} \mathrm{d} a \sin ^{-1}\left(\frac{2 a}{\sqrt{+}+\sqrt{-}}\right) a K_{0}\left(a / R_{\mathrm{d}}\right)
$$

where $\zeta(z)$ is the expression inside the parentheses in eq. (6.2), $\sqrt{ \pm} \equiv \sqrt{\left(z-z^{\prime}\right)^{2}+(a \pm R)^{2}}$, and $K_{0}$ is the modified Bessel function.

Figure 18 shows the variance along the cylindrical radius for the two models. Almost everywhere, the spherical disk model results in an overestimation of the variance by at most about $30 \%$ (so the dispersion is overestimated by at most about $15 \%$ ). This means that predictions made of signals from Sommerfeld-enhanced annhilations will be conservative.

\section{Conclusion}

Using a self-consistent phase-space distribution for the galactic DM, we have considered the annihilation of DM particles where $\sigma v$ is an arbitrary function of the velocity.

We have found that, for models with Sommerfeld enhancement, annihilations in the center of the halo are boosted, not only because of the larger density predicted in N-body simulations, but also due to the smaller average velocity of the DM particles. Including the latter effect raises the annihilation fluxes from the galactic center, typically by a factor of $\sim 1-10$, reaching $\gtrsim 100$ when resonances are present. 


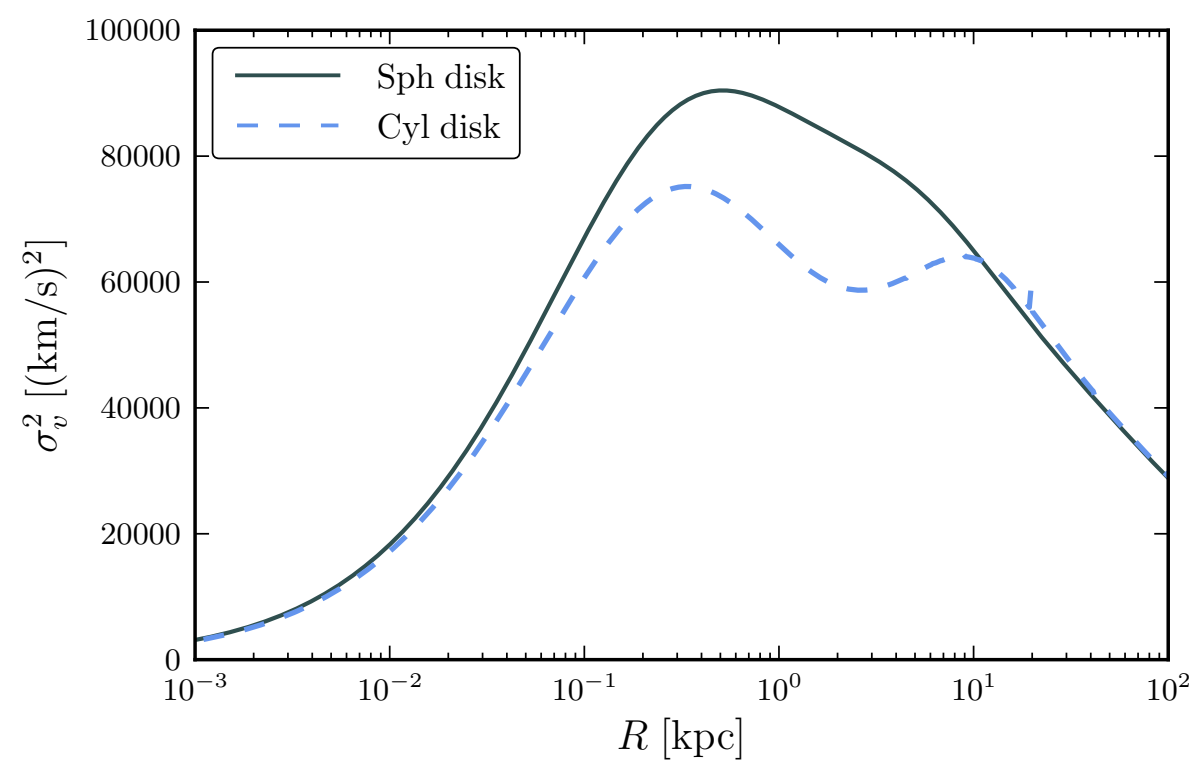

Figure 18. Variance of the DM particle velocity as a function of the distance from the center on the galactic plane. A cylindrical disk model is used in the first case; a spherical model is used in the latter. A NFW halo and a baryonic bulge is used in both cases.

As a consequence, the stringent constraints from synchrotron and gamma-ray emission on DM scenarios that attempt to explain the anomalies in the positron fraction observed at $\mathrm{GeV}$ energies are further strengthened.

\section{Acknowledgments}

The authors are grateful to Jim Buckley, Wyn Evans and Michael Ogilvie for useful conversations. This work was supported in part by the U.S. DOE under Contract No. DE-FG0291ER40628 and the NSF under Grant No. PHY-0855580.

\section{A Relative velocity distribution for an ergodic system}

Here, we write eq. (4.9) in terms of the individual phase-space distribution functions. Noting that eq. (4.9) is trivially zero when $v_{\text {rel }}=0$ or $v_{\text {rel }} \geq \sqrt{8 \tilde{\psi}}$, and that $\tilde{f}(\tilde{\epsilon})=0$ for $\tilde{\epsilon} \leq 0$, the integrand is non-zero when

$$
\begin{gathered}
0 \leq \tilde{v}_{\mathrm{cm}} \leq \sqrt{2 \tilde{\psi}}-\frac{\tilde{v}_{\text {rel }}}{2} \quad \text { and } \quad 0 \leq z \leq 1 \\
\text { or } \\
\sqrt{2 \tilde{\psi}}-\frac{\tilde{v}_{\mathrm{rel}}}{2} \leq \tilde{v}_{\mathrm{cm}} \leq \frac{\sqrt{8 \tilde{\psi}-\tilde{v}_{\mathrm{rel}}}}{2} \quad \text { and } \quad 0 \leq z \leq \frac{8 \tilde{\psi}-\tilde{v}_{\mathrm{rel}}^{2}-4 \tilde{v}_{\mathrm{cm}}^{2}}{4 \tilde{v}_{\mathrm{cm}} \tilde{v}_{\mathrm{rel}}}
\end{gathered}
$$


When $\tilde{v}_{\text {rel }}=0$, eq. (4.9) is trivially zero. Otherwise,

$$
\begin{aligned}
& P_{r, \text { rel }}\left(v_{\text {rel }}\right)=\frac{2 \tilde{v}_{\text {rel }}^{2}}{\pi^{2} \tilde{\rho}(r)^{2}}\left(\frac{r_{\text {vir }}}{G M_{\text {vir }}}\right)^{3} \times \\
& \left\{\int_{0}^{\sqrt{2 \tilde{\psi}}-\frac{\tilde{v}_{\mathrm{rel}}}{2}} \mathrm{~d} \tilde{v}_{\mathrm{cm}} \tilde{v}_{\mathrm{cm}}^{2} \int_{0}^{1} \mathrm{~d} z+\int_{\frac{\sqrt{2 \tilde{\psi}-\tilde{v}_{\mathrm{rel}}}}{2}}^{\frac{\sqrt{8 \tilde{\psi}-\tilde{v}_{\mathrm{rel}}^{2}}}{2}} \mathrm{~d} \tilde{v}_{\mathrm{cm}} \tilde{v}_{\mathrm{cm}}^{2} \int_{0}^{\frac{8 \tilde{\psi}-\tilde{v}_{\mathrm{rel}}^{2}-4 \tilde{v}_{\mathrm{cm}}^{2}}{4 \tilde{v}_{\mathrm{rm}}}} \mathrm{d} z\right\} \times \\
& \tilde{f}\left(\tilde{\psi}(r)-\frac{\tilde{v}_{\mathrm{cm}}^{2}}{2}-\frac{\tilde{v}_{\mathrm{rel}}^{2}}{8}-\frac{\tilde{v}_{\mathrm{cm}} \tilde{v}_{\mathrm{rel}} z}{2}\right) \tilde{f}\left(\tilde{\psi}(r)-\frac{\tilde{v}_{\mathrm{cm}}^{2}}{2}-\frac{\tilde{v}_{\mathrm{rel}}^{2}}{8}+\frac{\tilde{v}_{\mathrm{cm}} \tilde{v}_{\mathrm{rel}} z}{2}\right) .
\end{aligned}
$$

\section{References}

[1] G. Jungman, M. Kamionkowski, and K. Griest, Supersymmetric dark matter, Phys.Rept. 267 (1996) 195-373, [hep-ph/9506380].

[2] G. Bertone, D. Hooper, and J. Silk, Particle dark matter: Evidence, candidates and constraints, Phys.Rept. 405 (2005) 279-390, [hep-ph/0404175].

[3] J. L. Newstead, T. D. Jacques, L. M. Krauss, J. B. Dent, and F. Ferrer, The Scientific Reach of Multi-Ton Scale Dark Matter Direct Detection Experiments, arXiv:1306.3244.

[4] J. Ellis, K. A. Olive, and P. Sandick, Update on the Direct Detection of Dark Matter in MSSM Models with Non-Universal Higgs Masses, New J.Phys. 11 (2009) 105015, [arXiv:0905.0107].

[5] K. Freese, M. Lisanti, and C. Savage, Annual Modulation of Dark Matter: A Review, arXiv: 1209.3339.

[6] DAMA Collaboration, LIBRA Collaboration Collaboration, R. Bernabei et al., New results from DAMA/LIBRA, Eur.Phys.J. C67 (2010) 39-49, [arXiv:1002.1028].

[7] CoGeNT Collaboration Collaboration, C. Aalseth et al., CoGeNT: A Search for Low-Mass Dark Matter using p-type Point Contact Germanium Detectors, arXiv:1208.5737.

[8] CDMS Collaboration Collaboration, R. Agnese et al., Dark Matter Search Results Using the Silicon Detectors of CDMS II, Phys.Rev.Lett. (2013) [arXiv:1304.4279].

[9] XENON100 Collaboration Collaboration, E. Aprile et al., Dark Matter Results from 225 Live Days of XENON100 Data, Phys.Rev.Lett. 109 (2012) 181301, [arXiv:1207.5988].

[10] CDMS-II Collaboration Collaboration, Z. Ahmed et al., Results from a Low-Energy Analysis of the CDMS II Germanium Data, Phys.Rev.Lett. 106 (2011) 131302, [arXiv: 1011.2482].

[11] M. T. Frandsen, F. Kahlhoefer, C. McCabe, S. Sarkar, and K. Schmidt-Hoberg, The unbearable lightness of being: CDMS versus XENON, arXiv:1304.6066.

[12] D. Tucker-Smith and N. Weiner, Inelastic dark matter, Phys.Rev. D64 (2001) 043502, [hep-ph/0101138].

[13] A. Kurylov and M. Kamionkowski, Generalized analysis of weakly interacting massive particle searches, Phys.Rev. D69 (2004) 063503, [hep-ph/0307185].

[14] J. L. Feng, J. Kumar, D. Marfatia, and D. Sanford, Isospin-Violating Dark Matter, Phys.Lett. B703 (2011) 124-127, [arXiv:1102.4331].

[15] C. Savage, G. Gelmini, P. Gondolo, and K. Freese, XENON10/100 dark matter constraints in comparison with CoGeNT and DAMA: examining the Leff dependence, Phys.Rev. D83 (2011) 055002, [arXiv: 1006.0972]. 
[16] C. Kelso, D. Hooper, and M. R. Buckley, Toward A Consistent Picture For CRESST, CoGeNT and DAMA, Phys.Rev. D85 (2012) 043515, [arXiv:1110.5338].

[17] C. McCabe, The Astrophysical Uncertainties Of Dark Matter Direct Detection Experiments, Phys.Rev. D82 (2010) 023530, [arXiv: 1005.0579].

[18] M. T. Frandsen, F. Kahlhoefer, C. McCabe, S. Sarkar, and K. Schmidt-Hoberg, Resolving astrophysical uncertainties in dark matter direct detection, JCAP 1201 (2012) 024, [arXiv: 1111.0292].

[19] A. M. Green, Astrophysical uncertainties on direct detection experiments, Mod.Phys.Lett. A27 (2012) 1230004, [arXiv:1112.0524].

[20] M. Fairbairn, T. Douce, and J. Swift, Quantifying Astrophysical Uncertainties on Dark Matter Direct Detection Results, arXiv:1206.2693.

[21] P. Ullio and M. Kamionkowski, Velocity distributions and annual modulation signatures of weakly interacting massive particles, JHEP 0103 (2001) 049, [hep-ph/0006183].

[22] M. Lisanti, L. E. Strigari, J. G. Wacker, and R. H. Wechsler, The Dark Matter at the End of the Galaxy, Phys.Rev. D83 (2011) 023519, [arXiv:1010.4300].

[23] M. Vogelsberger, A. Helmi, V. Springel, S. D. White, J. Wang, et al., Phase-space structure in the local dark matter distribution and its signature in direct detection experiments, Mon.Not.Roy.Astron.Soc. 395 (2009) 797-811, [arXiv:0812.0362].

[24] P. Belli, R. Cerulli, N. Fornengo, and S. Scopel, Effect of the galactic halo modeling on the DAMA / NaI annual modulation result: an Extended analysis of the data for WIMPs with a purely spin independent coupling, Phys.Rev. D66 (2002) 043503, [hep-ph/0203242].

[25] R. Catena and P. Ullio, The local dark matter phase-space density and impact on WIMP direct detection, JCAP 1205 (2012) 005, [arXiv:1111.3556].

[26] P. J. Fox, G. D. Kribs, and T. M. Tait, Interpreting Dark Matter Direct Detection Independently of the Local Velocity and Density Distribution, Phys.Rev. D83 (2011) 034007, [arXiv:1011.1910].

[27] J. Knodlseder, V. Lonjou, P. Jean, M. Allain, P. Mandrou, et al., Early SPI / INTEGRAL contraints on the morphology of the $511 \mathrm{keV}$ line emission in the 4 th galactic quadrant, Astron.Astrophys. 411 (2003) L457-L460, [astro-ph/0309442].

[28] M. Su, T. R. Slatyer, and D. P. Finkbeiner, Giant Gamma-ray Bubbles from Fermi-LAT: AGN Activity or Bipolar Galactic Wind?, Astrophys.J. 724 (2010) 1044-1082, [arXiv:1005.5480].

[29] T. Bringmann, X. Huang, A. Ibarra, S. Vogl, and C. Weniger, Fermi LAT Search for Internal Bremsstrahlung Signatures from Dark Matter Annihilation, JCAP 1207 (2012) 054, [arXiv: 1203.1312].

[30] C. Weniger, A Tentative Gamma-Ray Line from Dark Matter Annihilation at the Fermi Large Area Telescope, JCAP 1208 (2012) 007, [arXiv:1204.2797].

[31] PAMELA Collaboration Collaboration, O. Adriani et al., An anomalous positron abundance in cosmic rays with energies 1.5-100 GeV, Nature 458 (2009) 607-609, [arXiv:0810.4995].

[32] AMS Collaboration Collaboration, M. Aguilar et al., First Result from the Alpha Magnetic Spectrometer on the International Space Station: Precision Measurement of the Positron Fraction in Primary Cosmic Rays of 0.5350 GeV, Phys.Rev.Lett. 110 (2013), no. 14141102.

[33] C. Boehm, D. Hooper, J. Silk, M. Casse, and J. Paul, MeV dark matter: Has it been detected?, Phys.Rev.Lett. 92 (2004) 101301, [astro-ph/0309686].

[34] D. Hooper, F. Ferrer, C. Boehm, J. Silk, J. Paul, et al., Possible evidence for MeV dark matter in dwarf spheroidals, Phys.Rev.Lett. 93 (2004) 161302, [astro-ph/0311150]. 
[35] D. Hooper and T. R. Slatyer, Two Emission Mechanisms in the Fermi Bubbles: A Possible Signal of Annihilating Dark Matter, arXiv:1302.6589.

[36] D. Hooper and T. Linden, On The Origin Of The Gamma Rays From The Galactic Center, Phys.Rev. D84 (2011) 123005, [arXiv:1110.0006].

[37] K. N. Abazajian and M. Kaplinghat, Detection of a Gamma-Ray Source in the Galactic Center Consistent with Extended Emission from Dark Matter Annihilation and Concentrated Astrophysical Emission, Phys.Rev. D86 (2012) 083511, [arXiv:1207.6047].

[38] M. Kuhlen, M. Vogelsberger, and R. Angulo, Numerical Simulations of the Dark Universe: State of the Art and the Next Decade, Phys.Dark Univ. 1 (2012) 50-93, [arXiv:1209.5745].

[39] C. Boehm, T. Ensslin, and J. Silk, Can Annihilating dark matter be lighter than a few GeVs?, J.Phys. G30 (2004) 279-286, [astro-ph/0208458].

[40] C. Boehm and P. Fayet, Scalar dark matter candidates, Nucl.Phys. B683 (2004) 219-263, [hep-ph/0305261].

[41] D. Hooper, A. Stebbins, and K. M. Zurek, Excesses in cosmic ray positron and electron spectra from a nearby clump of neutralino dark matter, Phys.Rev. D79 (2009) 103513, [arXiv:0812.3202].

[42] L. Pieri, J. Lavalle, G. Bertone, and E. Branchini, Implications of High-Resolution Simulations on Indirect Dark Matter Searches, Phys.Rev. D83 (2011) 023518, [arXiv:0908.0195].

[43] J. Hisano, S. Matsumoto, M. M. Nojiri, and O. Saito, Non-perturbative effect on dark matter annihilation and gamma ray signature from galactic center, Phys.Rev. D71 (2005) 063528, [hep-ph/0412403].

[44] N. Arkani-Hamed, D. P. Finkbeiner, T. R. Slatyer, and N. Weiner, A Theory of Dark Matter, Phys.Rev. D79 (2009) 015014, [arXiv:0810.0713].

[45] M. Pospelov and A. Ritz, Astrophysical Signatures of Secluded Dark Matter, Phys.Lett. B671 (2009) 391-397, [arXiv:0810.1502].

[46] M. Cirelli, A. Strumia, and M. Tamburini, Cosmology and Astrophysics of Minimal Dark Matter, Nucl.Phys. B787 (2007) 152-175, [arXiv:0706.4071].

[47] F. Donato, D. Maurin, P. Brun, T. Delahaye, and P. Salati, Constraints on WIMP Dark Matter from the High Energy PAMELA $\bar{p} / p$ data, Phys.Rev.Lett. 102 (2009) 071301, [arXiv: 0810.5292].

[48] G. Bertone, M. Cirelli, A. Strumia, and M. Taoso, Gamma-ray and radio tests of the e+eexcess from DM annihilations, JCAP 0903 (2009) 009, [arXiv:0811.3744].

[49] L. Bergstrom, G. Bertone, T. Bringmann, J. Edsjo, and M. Taoso, Gamma-ray and Radio Constraints of High Positron Rate Dark Matter Models Annihilating into New Light Particles, Phys.Rev. D79 (2009) 081303, [arXiv:0812.3895].

[50] M. Cirelli, P. Panci, and P. D. Serpico, Diffuse gamma ray constraints on annihilating or decaying Dark Matter after Fermi, Nucl.Phys. B840 (2010) 284-303, [arXiv:0912.0663].

[51] A. De Simone, A. Riotto, and W. Xue, Interpretation of AMS-02 Results: Correlations among Dark Matter Signals, JCAP 1305 (2013) 003, [arXiv:1304.1336].

[52] N. Evans, F. Ferrer, and S. Sarkar, A 'Baedecker' for the dark matter annihilation signal, Phys.Rev. D69 (2004) 123501, [astro-ph/0311145].

[53] Y. Ascasibar, P. Jean, C. Boehm, and J. Knoedlseder, Constraints on dark matter and the shape of the Milky Way dark halo from the 511-keV line, Mon.Not.Roy.Astron.Soc. 368 (2006) 1695-1705, [astro-ph/0507142]. 
[54] S. Campbell and B. Dutta, Effects of P-wave Annihilation on the Angular Power Spectrum of Extragalactic Gamma-rays from Dark Matter Annihilation, Phys.Rev. D84 (2011) 075004, [arXiv:1106.4621].

[55] B. Robertson and A. Zentner, Dark Matter Annihilation Rates with Velocity-Dependent Annihilation Cross Sections, Phys.Rev. D79 (2009) 083525, [arXiv:0902.0362].

[56] J. Khoury, Theories of Dark Energy with Screening Mechanisms, arXiv:1011.5909.

[57] J. Bernstein, Kinetic theory in the expanding universe. Cambridge University Press, 1988.

[58] M. Banados, J. Silk, and S. M. West, Kerr Black Holes as Particle Accelerators to Arbitrarily High Energy, Phys.Rev.Lett. 103 (2009) 111102, [arXiv:0909.0169].

[59] L. Sadeghian, F. Ferrer, and C. M. Will, Dark matter distributions around massive black holes: A general relativistic analysis, arXiv:1305.2619.

[60] J. Binney and S. Tremaine, Galactic Dynamics: Second Edition. Princeton University Press, 2008.

[61] A. Drukier, K. Freese, and D. Spergel, Detecting Cold Dark Matter Candidates, Phys.Rev. D33 (1986) 3495-3508.

[62] J. Diemand and B. Moore, The structure and evolution of cold dark matter halos, Adv.Sci.Lett. 4 (2011) 297-310, [arXiv:0906.4340].

[63] C. S. Frenk and S. D. White, Dark matter and cosmic structure, Annalen Phys. 524 (2012) 507-534, [arXiv: 1210.0544].

[64] W. de Blok, The Core-Cusp Problem, Adv.Astron. 2010 (2010) 789293, [arXiv:0910.3538].

[65] W. Dehnen, A Family of Potential-Density Pairs for Spherical Galaxies and Bulges, Mon.Not.Roy.Astron.Soc. 265 (1993) 250.

[66] J. F. Navarro, C. S. Frenk, and S. D. White, A Universal density profile from hierarchical clustering, Astrophys.J. 490 (1997) 493-508, [astro-ph/9611107].

[67] A. Deason, V. Belokurov, N. Evans, S. Koposov, R. Cooke, et al., The cold veil of the Milky Way stellar halo, arXiv:1205.6203.

[68] L. E. Strigari, J. S. Bullock, M. Kaplinghat, J. D. Simon, M. Geha, et al., A common mass scale for satellite galaxies of the Milky Way, Nature 454 (2008) 1096-1097, [arXiv:0808.3772].

[69] M. G. Walker, Dark Matter in the Milky Way's Dwarf Spheroidal Satellites, arXiv:1205.0311.

[70] A. V. Macciò, A. A. Dutton, and F. C. van den Bosch, Concentration, spin and shape of dark matter haloes as a function of the cosmological model: WMAP1, WMAP3 and WMAP5 results, MNRAS 391 (Dec., 2008) 1940-1954, [arXiv:0805.1926].

[71] V. F. Cardone, E. Piedipalumbo, and C. Tortora, Spherical galaxy models with power law logarithmic slope, Mon.Not.Roy.Astron.Soc. 358 (2005) 1325-1336, [astro-ph/0501151].

[72] J. F. Navarro, E. Hayashi, C. Power, A. Jenkins, C. S. Frenk, et al., The Inner structure of Lambda-CDM halos 3: Universality and asymptotic slopes, Mon.Not.Roy.Astron.Soc. $\mathbf{3 4 9}$ (2004) 1039, [astro-ph/0311231].

[73] A. W. Graham, D. Merritt, B. Moore, J. Diemand, and B. Terzic, Empirical models for Dark Matter Halos. I. Nonparametric Construction of Density Profiles and Comparison with Parametric Models, Astron.J. 132 (2006) 2685-2700, [astro-ph/0509417].

[74] A. R. Duffy, J. Schaye, S. T. Kay, and C. Dalla Vecchia, Dark matter halo concentrations in the Wilkinson Microwave Anisotropy Probe year 5 cosmology, Mon.Not.Roy.Astron.Soc. 390 (2008) L64, [arXiv:0804.2486].

[75] M. G. Walker and J. Penarrubia, A Method for Measuring (Slopes of) the Mass Profiles of Dwarf Spheroidal Galaxies, Astrophys.J. 742 (2011) 20, [arXiv:1108.2404]. 
[76] N. Amorisco, A. Agnello, and N. Evans, The core size of the Fornax dwarf Spheroidal, arXiv: 1210.3157.

[77] A. Burkert, The Structure of dark matter halos in dwarf galaxies, IAU Symp. 171 (1996) 175, [astro-ph/9504041].

[78] P. Salucci, M. I. Wilkinson, M. G. Walker, G. F. Gilmore, E. K. Grebel, et al., Dwarf spheroidal galaxy kinematics and spiral galaxy scaling laws, arXiv:1111.1165.

[79] L. E. Strigari and R. Trotta, Reconstructing WIMP Properties in Direct Detection Experiments Including Galactic Dark Matter Distribution Uncertainties, JCAP 0911 (2009) 019, [arXiv:0906.5361].

[80] M. Kuhlen, N. Weiner, J. Diemand, P. Madau, B. Moore, et al., Dark Matter Direct Detection with Non-Maxwellian Velocity Structure, JCAP 1002 (2010) 030, [arXiv:0912.2358].

[81] Y.-Y. Mao, L. E. Strigari, R. H. Wechsler, H.-Y. Wu, and O. Hahn, Halo-to-Halo Similarity and Scatter in the Velocity Distribution of Dark Matter, Astrophys.J. 764 (2013) 35, [arXiv:1210.2721].

[82] S. Chaudhury, P. Bhattacharjee, and R. Cowsik, Direct detection of WIMPs : Implications of a self-consistent truncated isothermal model of the Milky Way's dark matter halo, JCAP 1009 (2010) 020, [arXiv: 1006.5588].

[83] F. Ferrer and D. R. Hunter. Work in progress.

[84] M. Kuhlen, P. Madau, and J. Silk, Exploring Dark Matter with Milky Way substructure, Science 325 (2009) 970-973, [arXiv:0907.0005].

[85] J. Magorrian and J. Binney, Predicting line-of-sight velocity distributions of elliptical galaxies, MNRAS 271 (Dec., 1994) 949.

[86] http://www.fysik.su.se/ edsjo/darksusy/.

[87] P. Gondolo, Either neutralino dark matter or cuspy dark halos, Phys.Lett. B494 (2000) 181-186, [hep-ph/0002226].

[88] M. Cirelli and P. Panci, Inverse Compton constraints on the Dark Matter e+e-excesses, Nucl.Phys. B821 (2009) 399-416, [arXiv:0904.3830].

[89] R. Iengo, Sommerfeld enhancement: general results from field theory diagrams, Journal of High Energy Physics 5 (May, 2009) 24, [arXiv:0902.0688].

[90] S. Cassel, Sommerfeld factor for arbitrary partial wave processes, J.Phys.G G37 (2010) 105009, [arXiv:0903.5307].

[91] T. R. Slatyer, The Sommerfeld enhancement for dark matter with an excited state, JCAP 1002 (2010) 028, [arXiv:0910.5713].

[92] J. L. Feng, M. Kaplinghat, and H.-B. Yu, Sommerfeld Enhancements for Thermal Relic Dark Matter, Phys.Rev. D82 (2010) 083525, [arXiv:1005.4678].

[93] http://fermi.gsfc.nasa.gov/.

[94] E. Visbal, A. Loeb, and L. Hernquist, A Simple Model for the Density Profiles of Isolated Dark Matter Halos, arXiv:1206.5852.

[95] We thank Yao-Yuan Mao for pointing this out.

[96] M. Baes and H. Dejonghe, The Hernquist model revisited: Completely analytical anisotropic dynamical models, Astron.Astrophys. 393 (2002) 485-498, [astro-ph/0207233].

[97] T. R. Slatyer, N. Toro, and N. Weiner, Sommerfeld-enhanced annihilation in dark matter substructure: Consequences for constraints on cosmic-ray excesses, Phys.Rev. D86 (2012) 083534, [arXiv:1107.3546]. 
[98] I. Cholis and L. Goodenough, Consequences of a Dark Disk for the Fermi and PAMELA Signals in Theories with a Sommerfeld Enhancement, JCAP 1009 (2010) 010, [arXiv: 1006.2089].

[99] D. Lynden-Bell, Stellar dynamics: Exact solution of the self-gravitation equation, MNRAS 123 (1962) 447. 